\title{
All-electron Formalism for Total Energy Strain Derivatives and Stress Tensor Components for Numeric Atom-Centered Orbitals
}

\author{
Franz Knuth $^{\mathrm{a}}$, Christian Carbogno ${ }^{\mathrm{a}, *}$, Viktor Atalla ${ }^{\mathrm{a}}$, Volker Blum ${ }^{\mathrm{a}, \mathrm{b}}$, Matthias Scheffler ${ }^{\mathrm{a}}$ \\ ${ }^{a}$ Fritz-Haber-Institut der Max-Planck-Gesellschaft, Faradayweg 4-6, D-14195 Berlin, Germany \\ ${ }^{b}$ Department of Materials Science and Mechanical Engineering, Duke University, Durham, NC 27708, USA
}

\begin{abstract}
We derive and implement the strain derivatives of the total energy of solids, i.e., the analytic stress tensor components, in an all-electron, numeric atom-centered orbital based density-functional formalism. We account for contributions that arise in the semilocal approximation (LDA/GGA) as well as in the generalized Kohn-Sham case, in which a fraction of exact exchange (hybrid functionals) is included. In this work, we discuss the details of the implementation including the numerical corrections for sparse integrations grids which allow to produce accurate results. We validate the implementation for a variety of test cases by comparing to strain derivatives performed via finite differences. Additionally, we include the detailed definition of the overlapping atom-centered integration formalism used in this work to obtain total energies and their derivatives.
\end{abstract}

Keywords: Stress tensor, Strain derivatives, Density-functional theory, Hybrid functionals, Atom-centered basis functions PACS: 71.15.-m

\section{Introduction}

Density-functional theory (DFT) enjoys high popularity as an $a b$ initio formalism to compute the ground state electron density and energy of molecules, clusters, and solids. As formalized in the Hellmann-Feynman theorem and generalized in the $2 n+1$ theorem [1], the so-called atomic forces, i.e., the derivatives of the Born-Oppenheimer potential-energy surface with respect to the nuclear coordinates, can be derived as analytical expressions. Care must be taken since correction can terms arise, e.g., Pulay terms [2] if the basis functions move with the atoms. The atomic forces enable efficient DFT-based structure optimization algorithms that allow to determine the local minima of the potential-energy surface associated with the electronic ground state. By these means, one can identify the stable and metastable geometries at zero Kelvin, which in turn provide the founding for further computational investigations, e.g., via first-principles atomistic thermodynamics [3]. To apply such structure optimization methods to materials modeled as periodic solids, one must additionally take into account the lattice degrees of freedom and the respective derivatives of the energy with respect to strain, i.e., the stress.

The strain tensor $\varepsilon$ describes the elastic deformation of a crystal relative to a reference state. This corresponds to a transformation of all real space coordinates and its derived quantities, e.g., the atomic positions $\boldsymbol{R}$

$$
R_{\alpha}(\varepsilon)=\sum_{\beta}\left(\delta_{\alpha \beta}+\varepsilon_{\alpha \beta}\right) R_{\beta}(0)
$$

\footnotetext{
${ }^{*}$ Corresponding author

Email address: carbogno@fhi-berlin.mpg.de (Christian Carbogno)
}

with respect to the zero strain reference $\boldsymbol{R}(0), \alpha, \beta=1,2,3$ denote the Cartesian components. In general, Greek indices stand for the three Cartesian components throughout this paper. For a unit cell with volume $V$, the stress tensor $\sigma$ is defined [4, 5] as the first order change in the total (Born-Oppenheimer) energy of the unit cell $E_{\text {tot }}$ under a symmetrical, infinitesimal strain deformation $\varepsilon$

$$
\sigma_{\lambda \mu}=\left.\frac{1}{V} \frac{\partial E_{\mathrm{tot}}}{\partial \varepsilon_{\lambda \mu}}\right|_{\varepsilon=0} .
$$

Given that only symmetric strain deformations are used in the definition of the stress tensor, the stress tensor is symmetric as well and thus consists of six independent entries. Although this definition is unique, a practical implementation of these derivatives depends very much on the numerical details chosen for the DFT formalism, e.g., on the basis set type.

Nielsen and Martin [6, 7] first demonstrated that the stress can indeed be efficiently and accurately assessed in a DFT framework. For this purpose, they employed a plane wave basis set expansion (together with norm-conserving pseudopotentials) and the local-density approximation. Later works extended the stress tensor implementation for plane waves to ultra-soft pseudopotentials [8] including the contributions of the generalized orthonormality condition. Thonhauser and coworkers [9] as well as Nagasako and coworkers [5] summarized the strain derivatives for the linearized augmented plane wave method. The former derived a surface term due to the discontinuities at the boundaries between muffin tins and interstitial region and latter presented a correction because the number of plane waves in the interstitial region changes under strain. Kresse and coworkers [10] as well as Torrent and coworkers [11] derived the stress tensor for the projector augmented- 
wave (PAW) method and discussed the additional terms arising from the compensating charges of the PAW method. Kudin and coworkers [12] discussed the implementation of the stress tensor for Gaussian-type orbitals (GTO) while evaluating the electrostatic contributions entirely in real space using a fast multipole method. Doll and coworkers [13, 14] employed GTOs, too, including the strain derivatives for Hartree-Fock calculations. Furthermore, Soler and coworkers [15] presented the strain derivatives for numeric atom-centered orbitals with norm-conserving pseudopotentials calculating the electrostatics by fast Fourier transforms.

In this paper, we derive the strain derivatives, i.e., the analytic stress tensor components, in an all-electron, numeric atom-centered orbital based density-functional formalism. We discuss our implementation of these formulae in the electronic structure theory code FHI-aims [16] including strain derivatives of hybrid functionals and the van der Waals (vdW) correction scheme of Tkatchenko and Scheffler [17]. Hereby, all electrostatic contributions are evaluated in real space using a multipole expansion including Ewald's method [18] together with corrections. Furthermore, we account for Pulay terms due to our atom-centered basis functions.

The remainder of the paper is organized as follows: In Sec.2, the general mathematical formalism of DFT and of the analytical stress tensor are presented. Sec. 3 gives a detailed derivation of the various contributions that arise in a numeric atomcentered orbital based DFT formalism. Details of the numerical implementation are discussed in Sec. 4. Finally in Sec. 5, we investigate the accuracy and the efficiency of our implementation by comparing our analytical stress tensor components to strain derivatives computed via finite differences. We compare these two quantities for a wide range of systems that range from metals via semiconductors and insulators to organic crystals to demonstrate the general validity of our implementation across the periodic table. Furthermore, we also study the behavior of our implementation for different exchange-correlation functionals, basis set sizes, system sizes, and integration grids to investigate the convergence behavior of the analytical stress tensor. The computational performance of our implementation is discussed in as well. For the sake of completeness, Sec.6 gives an overview on how the stress tensor can be used to optimize the unit cell of a crystal (i.e., finding energy minima) under external pressure.

\section{Formalism}

\subsection{Total energy in DFT}

In Kohn-Sham (KS) DFT, the total energy of a system at given nuclear configuration reads [19, 20]

$$
E_{\mathrm{tot}}=E_{\mathrm{KS}}\left[n_{0}\right]+E_{\mathrm{nuc}}
$$

with the ground state electron density $n_{0}$, the nuclear-nuclear energy $E_{\text {nuc }}$, and the Kohn-Sham energy functional

$$
E_{\mathrm{KS}}[n]=T_{\mathrm{s}}[n]+E_{\mathrm{ext}}[n]+E_{\mathrm{H}}[n]+E_{\mathrm{xc}}[n] .
$$

$T_{\mathrm{s}}$ is the kinetic energy functional of non-interacting electrons, $E_{\text {ext }}$ the electron-nuclear energy, $E_{\mathrm{H}}$ the Hartree energy, and $E_{\mathrm{xc}}$ the exchange-correlation energy functional. For clarity, we avoid an explicitly spin-polarized notation, a formal generalization to collinear (scalar) spin-DFT is straightforward and in fact included in the implementation. Also, it is important to note that the formally correct separation of the electrostatic interactions in $E_{\text {nuc }}, E_{\text {ext }}$, and $E_{\mathrm{H}}$ is computationally not possible in periodic systems, as discussed in more detail in Sec. 3.4

The ground state electron density for fixed nuclear coordinates is obtained by solving the variational equation for the electron density $n$,

$$
\delta\left[E_{\mathrm{KS}}[n]-\mu\left(\int d \boldsymbol{r} n(\boldsymbol{r})-N_{\mathrm{e}}\right)\right]=0,
$$

with the chemical potential $\mu=\delta E_{\mathrm{KS}} / \delta n$ and the number of electrons $N_{\mathrm{e}}$. This yields the Kohn-Sham single particle equation [20],

$$
\hat{h}_{\mathrm{KS}}\left|\psi_{i}\right\rangle=\epsilon_{i}\left|\psi_{i}\right\rangle
$$

the solution of which yields the Kohn-Sham orbitals $\psi_{i}$ and the corresponding eigenvalues $\epsilon_{i}$. The electron density is

$$
n(\boldsymbol{r})=\sum_{i} f_{i}\left|\psi_{i}(\boldsymbol{r})\right|^{2}
$$

in which $f_{i}$ denotes the occupation number of the orbitals. Furthermore,

$$
\hat{h}_{\mathrm{KS}}=\hat{t}_{\mathrm{S}}+\hat{v}_{\mathrm{ext}}+\hat{v}_{\mathrm{H}}[n]+\hat{v}_{\mathrm{xc}}[n]
$$

is the Kohn-Sham Hamiltonian. $\hat{t}_{\mathrm{s}}$ is the kinetic operator, $\hat{v}_{\mathrm{ext}}$ the electron-nuclear potential, $\hat{v}_{\mathrm{H}}$ the Hartree potential, and $\hat{v}_{\mathrm{xc}}$ the exchange-correlation potential.

In practice, Eq. (6) is solved by expanding the Kohn-Sham orbitals $\psi_{i}$ in a given basis set

$$
\psi_{i}(\boldsymbol{r})=\sum_{j} c_{i j} \varphi_{j}(\boldsymbol{r})
$$

with the expansion coefficients $c_{i j}$ and the basis functions $\varphi_{j}(\boldsymbol{r})$, which leads to a generalized eigenvalue problem of the form

$$
\sum_{j}\left\langle\varphi_{i}\left|\hat{h}_{\mathrm{KS}}\right| \varphi_{j}\right\rangle c_{j l}=\epsilon_{l} \sum_{j}\left\langle\varphi_{i} \mid \varphi_{j}\right\rangle c_{j l} .
$$

Here, $\langle. \mid$.$\rangle denotes the usual bra-ket notation for the inner prod-$ uct in Hilbert space (integral in real space). In the case of FHI-aims, the basis functions are real-valued atom-centered orbitals, i.e., they depend on the position of the atoms, and the basis set expansion takes the following explicit form

$$
\psi_{i}(\boldsymbol{r})=\sum_{j, J} c_{i j} \varphi_{j}\left(\boldsymbol{r}-\boldsymbol{R}_{J}\right)
$$

The sum runs over all atoms $J$ and basis functions $j$ which are associated with atom $J$, and $\boldsymbol{R}_{J}$ denotes the position of atom $J$. Accordingly, the density is a function of the expansion coefficients and thus Eq. (5) translates into a minimization of $E_{\mathrm{KS}}$ with respect to the expansion coefficients under the constraint of orthonormalized orbitals,

$$
E_{\mathrm{KS}}\left[n_{0}\right]=\min _{\left\{c_{i j}\right\}}\left[E_{\mathrm{KS}}-\sum_{i} f_{i} \epsilon_{i}\left(\left\langle\psi_{i} \mid \psi_{i}\right\rangle-1\right)\right] .
$$




\subsection{Fundamental formulae for strain derivatives}

\subsubsection{Properties of strain derivative}

The total energy derivative in Eq. (2) can be written as

$$
\left.\frac{\partial E_{\mathrm{tot}}}{\partial \varepsilon_{\lambda \mu}}\right|_{\varepsilon=0}=\left.\sum_{i} \frac{\partial E_{\mathrm{tot}}\left(u_{i}\right)}{\partial u_{i}} \frac{\partial u_{i}}{\partial \varepsilon_{\lambda \mu}}\right|_{\varepsilon=0},
$$

where we use the chain rule and the sum runs over all parameters $u_{i}$ that enter the total energy and change under a strain transformation. For specified numerical and computational settings (basis set, integration grids, and exchange-correlationfunctional), the total energy solely depends on the chemical species (mass, nuclear charge, and electronic configuration) and the positions of the individual atoms. Under periodic boundary conditions, the latter also includes the lattice vectors $\boldsymbol{a}_{n}$, since the periodic images of an atom with coordinate vector $\boldsymbol{R}_{I}$ are given by $\boldsymbol{R}_{I}^{\prime}=\boldsymbol{R}_{I}+\sum_{n} M_{n} \boldsymbol{a}_{n}$ with $M_{n}$ being integer numbers. Accordingly, the atomic positions, lattice vectors, basis set orbitals, and integration grid 1 are the only quantities changing under a strain transformation; the respective strain derivatives of the first two quantities are derived below. However, some contributions to the total energy do not depend directly on this set of parameters. Instead, they depend on derived quantities. These are the volume of the system and the reciprocal lattice $\boldsymbol{G}$-vectors. To achieve a more concise formalism later in this paper, the strain derivatives for these quantities are derived as well.

\subsubsection{Strain derivative of position vectors}

With Eq. (1), the strain derivative of a general position vector is

$$
\left.\frac{\partial r_{\alpha}}{\partial \varepsilon_{\lambda \mu}}\right|_{\varepsilon=0}=\sum_{\beta} \delta_{\alpha \lambda} \delta_{\beta \mu} r_{\beta}=\delta_{\alpha \lambda} r_{\mu}
$$

In many cases, we will encounter terms $f\left(\boldsymbol{r}_{A B}\right)$ that do not depend on the absolute position, but on position differences $\boldsymbol{r}_{A B}=$ $\boldsymbol{r}_{A}-\boldsymbol{r}_{B}$. With the chain rule, we get

$$
\begin{aligned}
\left.\frac{\partial f\left(\boldsymbol{r}_{A B}\right)}{\partial \varepsilon_{\lambda \mu}}\right|_{\varepsilon=0} & =\frac{\partial f\left(\boldsymbol{r}_{A B}\right)}{\partial r_{A, \lambda}} r_{\mathrm{A}, \mu}+\frac{\partial f\left(\boldsymbol{r}_{A B}\right)}{\partial r_{B, \lambda}} r_{B, \mu} \\
& =\frac{\partial f\left(\boldsymbol{r}_{A B}\right)}{\partial r_{A, \lambda}}\left(r_{A, \mu}-r_{B, \mu}\right) .
\end{aligned}
$$

Since the function $f\left(\boldsymbol{r}_{A B}\right)$ depends only on the difference $\boldsymbol{r}_{A}-\boldsymbol{r}_{B}$, the derivative in the second summand could be changed from $\frac{\partial}{\partial r_{B, \lambda}}$ to $-\frac{\partial}{\partial r_{A, \lambda}}$.

\subsubsection{Lattice vectors and strain derivative of volume}

Under a strain transformation, the lattice vectors $\boldsymbol{a}_{n}$ of the crystal transform in the same way as the position vectors (Eq. (1))

$$
a_{\alpha n}(\varepsilon)=\sum_{\beta}\left(\delta_{\alpha \beta}+\varepsilon_{\alpha \beta}\right) a_{\beta n}(0)
$$

\footnotetext{
${ }^{1}$ The atom-centered orbitals and the real space grid used for the electronic degrees of freedom move with the respective nuclear degrees of freedom so that their contributions to the strain derivatives need to be accounted for as well.
}

If we write the lattice vectors $\boldsymbol{a}_{n}$ as the columns of a matrix $A=$ $\left[\boldsymbol{a}_{1} \boldsymbol{a}_{2} \boldsymbol{a}_{3}\right]$ then the volume of the unit cell is given by

$$
V=|\operatorname{det} A| \text {. }
$$

With help of Eq. [16, we obtain the strained volume

$$
\begin{aligned}
V(\varepsilon) & =|\operatorname{det} A(\varepsilon)| \\
& =|\operatorname{det}(\mathbb{1}+\varepsilon)| V(0) \\
& =\left|\sum_{\alpha}\left(1+\varepsilon_{\alpha \alpha}\right)\right| V(0) .
\end{aligned}
$$

In the last step, the determinant was expanded up to first order in the strain. Taking the strain derivative of Eq. (18) leads to

$$
\left.\frac{\partial V}{\partial \varepsilon_{\lambda \mu}}\right|_{\varepsilon=0}=\sum_{\alpha} \delta_{\alpha \lambda} \delta_{\alpha \mu} V=\delta_{\lambda \mu} V
$$

\subsubsection{Strain derivative of reciprocal lattice vectors}

The unit vectors of the reciprocal lattice $\boldsymbol{b}_{n}$ can be written as the columns of a matrix $B=\left[\boldsymbol{b}_{1} \boldsymbol{b}_{2} \boldsymbol{b}_{3}\right]$ and are related to the lattice vectors by

$$
B=2 \pi\left(A^{\mathrm{T}}\right)^{-1} .
$$

Using the expression Eq. (16) for the strain transformation of lattice vectors, we get

$$
B(\varepsilon)=2 \pi\left(\mathbb{1}+\varepsilon^{\mathrm{T}}\right)^{-1}\left(A^{\mathrm{T}}\right)^{-1}=\left(\mathbb{1}-\varepsilon^{\mathrm{T}}\right) B(0),
$$

where we expanded the matrix inverse up to first order in the strain. For a unit vector of the reciprocal lattice $\boldsymbol{b}_{n}$, the component-wise notation of Eq. (21) is

$$
b_{\alpha n}(\varepsilon)=\sum_{\beta}\left(\delta_{\beta \alpha}-\varepsilon_{\beta \alpha}\right) b_{\beta n}(0)
$$

and thus we get the strain derivative

$$
\left.\frac{\partial b_{\alpha n}}{\partial \varepsilon_{\lambda \mu}}\right|_{\varepsilon=0}=-\sum_{\beta} \delta_{\beta \lambda} \delta_{\alpha \mu} b_{\beta n}=-\delta_{\alpha \mu} b_{\lambda n} .
$$

A general reciprocal lattice vector $\boldsymbol{G}$ is a linear combination of the three unit reciprocal lattice vectors

$$
\boldsymbol{G}=s_{1} \boldsymbol{b}_{1}+s_{2} \boldsymbol{b}_{2}+s_{3} \boldsymbol{b}_{3}
$$

with $s_{1}, s_{2}, s_{3}$ being integer numbers. Applying the result from Eq. (23) gives

$$
\left.\frac{\partial G_{\alpha}}{\partial \varepsilon_{\lambda \mu}}\right|_{\varepsilon=0}=-\delta_{\alpha \mu}\left(s_{1} b_{\lambda 1}+s_{2} b_{\lambda 2}+s_{3} b_{\lambda 3}\right)=-\delta_{\alpha \mu} G_{\lambda}
$$

Hence, for a quantity $f(\boldsymbol{G})$ that depends on the $\boldsymbol{G}$-vector, the strain derivative becomes

$$
\left.\frac{\partial f(\boldsymbol{G})}{\partial \varepsilon_{\lambda \mu}}\right|_{\varepsilon=0}=-\sum_{\alpha} \frac{\partial f(\boldsymbol{G})}{\partial G_{\alpha}} \delta_{\alpha \mu} G_{\lambda}=-\frac{\partial f(\boldsymbol{G})}{\partial G_{\mu}} G_{\lambda} .
$$


Integral over whole space

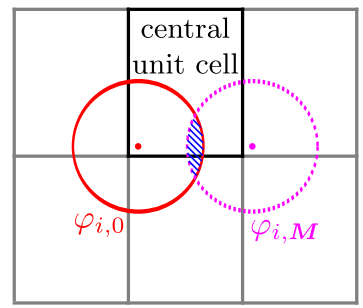

Integral over central unit cell

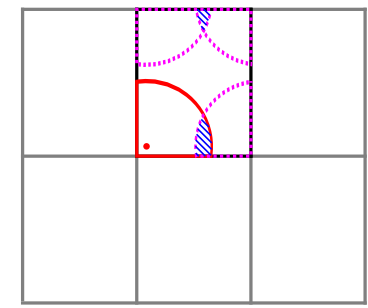

Figure 1: Illustration of two ways to calculate the integrals (blue stripes) between basis function $\varphi_{i, 0}$ (red, solid) centered at the corner of the central unit cell (black frame) and one of its periodic images $\varphi_{i, \boldsymbol{M}}$ (pink, dashed). The straightforward approach is to integrate both functions over the whole space to obtain the overlap integral as shown in the left picture. The integration can be separated into pieces lying inside individual unit cells which can be mapped into the central unit cell yielding the picture on the right hand side. There, the overlap integral is calculated by an integration over the central unit cell by considering those periodic images of the basis functions that extend into the central unit cell.

\subsubsection{Strain derivative of integrals over the unit cell volume}

In the case of periodic boundary conditions, Bloch-like generalized basis functions $\chi_{i, \boldsymbol{k}}(\boldsymbol{r})$ are defined. They are derived from the atom-centered basis function residing in different unit cells $\boldsymbol{M}, \varphi_{i, \boldsymbol{M}}(\boldsymbol{r})=\varphi_{i}\left(\boldsymbol{r}-\boldsymbol{R}_{I}-\boldsymbol{T}(\boldsymbol{M})\right)$. Here, $\boldsymbol{T}(\boldsymbol{M})=\boldsymbol{M} \cdot A$ yields the translation vector to a unit cell with $\boldsymbol{M}=\left(M_{1}, M_{2}, M_{3}\right)$ a vector with integer numbers and $A$ the matrix of the lattice vectors as introduced before. The generalized basis functions are (see Eq. 22 in Ref. [16])

$$
\chi_{i, \boldsymbol{k}}(\boldsymbol{r})=\sum_{\boldsymbol{M}} \exp (i \boldsymbol{k} \cdot \boldsymbol{T}(\boldsymbol{M})) \varphi_{i, \boldsymbol{M}}(\boldsymbol{r})
$$

Therefore, matrix elements like $h_{i j}=\left\langle\varphi_{i}\left|\hat{h}_{\mathrm{KS}}\right| \varphi_{j}\right\rangle$ from Eq. 10 become $\boldsymbol{k}$-dependent,

$$
h_{i j}(\boldsymbol{k})=\left\langle\chi_{i, \boldsymbol{k}}\left|\hat{h}_{\mathrm{Ks}}\right| \chi_{j, \boldsymbol{k}}\right\rangle .
$$

For calculations, the matrix elements per unit cell are needed, denoted with $\bar{h}_{i j}(\boldsymbol{k})$. Inserting Eq. (27) into Eq. (28) leads to two sums over the unit cells. Because of periodicity, they reduce to one sum for the matrix elements per unit cell,

$$
\bar{h}_{i j}(\boldsymbol{k})=\sum_{\boldsymbol{M}} \exp (i \boldsymbol{k} \cdot \boldsymbol{T}(\boldsymbol{M}))\left\langle\varphi_{i, 0}\left|\hat{h}_{\mathrm{KS}}\right| \varphi_{j, \boldsymbol{M}}\right\rangle
$$

with the index 0 denoting the central unit cell. The sum over the unit cells $\boldsymbol{M}$ is finite because all basis functions have a finite extension since they are bounded by a confinement potential [16].

The matrix elements in Eq.29) are integrals over the whole space. However, it is more convenient to integrate them in separate pieces with integrals over just one specific unit cell and then add up all contributions. Due to periodicity, all integrations can be performed in the central unit cell such that

$$
\sum_{\boldsymbol{M}}\left\langle\varphi_{i, 0}\left|\hat{h}_{\mathrm{KS}}\right| \varphi_{j, \boldsymbol{M}}\right\rangle=\sum_{\boldsymbol{M}, \boldsymbol{N}}^{\prime} \int_{V} \varphi_{i, \boldsymbol{N}}(\boldsymbol{r}) \hat{h}_{\mathrm{KS}} \varphi_{j, \boldsymbol{M}}(\boldsymbol{r})
$$

with $V$ the volume of the central unit cell. The sum $\sum_{M, N}^{\prime}$ runs only over those units cell that contain basis functions that have a non-zero contribution in the central unit cell. Fig. 1 illustrates the location of these integration volumes. With Eq. 30, Eq. 29) reads

$$
\bar{h}_{i j}(\boldsymbol{k})=\sum_{\boldsymbol{M}, \boldsymbol{N}}^{\prime} \exp (i \boldsymbol{k} \cdot[\boldsymbol{T}(\boldsymbol{N})-\boldsymbol{T}(\boldsymbol{M})]) \int_{V} \varphi_{i, \boldsymbol{N}}(\boldsymbol{r}) \hat{h}_{\mathrm{KS}} \varphi_{j, \boldsymbol{M}}(\boldsymbol{r})
$$

incorporating the Bloch phase factors.

As a consequence, the individual integral pieces of Eq. (31) change under strain since their boundary $V$ changes under strain, see Sec. 2.2.3, and this has to be accounted for. Let us consider the integral of an arbitrary function $f$ over the unit cell volume $V$

$$
I(V)=\int_{V} d \boldsymbol{r} f(\boldsymbol{r})
$$

In order to take the strain derivative of this expression, we need to change the integration variable to fractional coordinates $s$ and use the transformation theorem for integrals. The change of integration variables yields

$$
I(V)=V \int_{0}^{1} d s f(s)
$$

and the integration limits symbolize that the three components of the fractional coordinates lie in the range from 0 to 1 . Taking the strain derivative of this transformed integral results in

$$
\left.\frac{\partial I(V)}{\partial \varepsilon_{\lambda \mu}}\right|_{\varepsilon=0}=\delta_{\lambda \mu} I(V)+\left.\int_{V} d \boldsymbol{r} \frac{\partial f(\boldsymbol{r})}{\partial \varepsilon_{\lambda \mu}}\right|_{\varepsilon=0} .
$$

The first term arises due to the finite integration limits (which change under strain) and we will call it Jacobian term from here on.

\section{Strain derivative of total energy}

For the remainder of this paper, the notation is simplified by leaving out the explicit notation that the derivative is evaluated at the point $\varepsilon=0$.

\subsection{Kohn-Sham orbitals}

The strain derivative of the Kohn-Sham orbitals $\psi_{i}(\boldsymbol{r})$ can be calculated using Eq. (11) and (15)

$$
\frac{\partial \psi_{i}(\boldsymbol{r})}{\partial \varepsilon_{\lambda \mu}}=\sum_{j, J} c_{i j} \frac{\partial \varphi_{j}\left(\boldsymbol{r}-\boldsymbol{R}_{J}\right)}{\partial R_{J, \lambda}}\left(R_{J, \mu}-r_{\mu}\right)
$$

where the sum runs over all atoms $J$ and basis functions $j$ which are associated with atom $J$. The strain derivative of the gradient of an orbital can be done analogously

$$
\frac{\partial}{\partial \varepsilon_{\lambda \mu}} \frac{\partial \psi_{i}(\boldsymbol{r})}{\partial R_{K, v}}=\sum_{j, J} c_{i j} \frac{\partial^{2} \varphi_{j}\left(\boldsymbol{r}-\boldsymbol{R}_{J}\right)}{\partial R_{J, \lambda} \partial R_{K, v}}\left(R_{J, \mu}-r_{\mu}\right)
$$

which means that the Hessian of the basis functions is required for this strain derivative. 


\subsection{Normalization factor}

The strain derivative of the normalization factor of Eq. (12) yields

$$
\frac{\partial F_{\text {norm }}}{\partial \varepsilon_{\lambda \mu}}=-\delta_{\lambda \mu} \sum_{i} f_{i}\left\langle\psi_{i}\left|\epsilon_{i}\right| \psi_{i}\right\rangle-2 \sum_{i} f_{i}\left\langle\frac{\partial \psi_{i}}{\partial \varepsilon_{\lambda \mu}}\left|\epsilon_{i}\right| \psi_{i}\right\rangle .
$$

The inner product of the two Kohn-Sham orbitals yields two terms according to Eq. (34).

\subsection{Kinetic energy}

\subsubsection{Kinetic energy of non-interacting electrons}

The kinetic energy of non-interacting electrons in the nonrelativistic case is

$$
T_{\mathrm{s}}=\sum_{i} f_{i}\left\langle\psi_{i}\left|\hat{t}_{\mathrm{s}}\right| \psi_{i}\right\rangle
$$

with the kinetic energy operator $\hat{t}_{\mathrm{s}}=-\frac{1}{2} \Delta$. It is important that we consider the change of the Laplace operator under a strain transformation. The change of a position vector $\boldsymbol{r}$ under such a transformation is given by Eq. (1) and the respective inverse relation (up to first order in the strain) is $r_{\alpha}(0)=\sum_{\beta}\left(\delta_{\alpha \beta}-\right.$ $\left.\varepsilon_{\alpha \beta}\right) r_{\beta}(\varepsilon)$. Accordingly, the derivative with respect to positions transforms as

$$
\frac{\partial}{\partial r_{\alpha}(\varepsilon)}=\sum_{\beta} \frac{\partial r_{\beta}(0)}{\partial r_{\alpha}(\varepsilon)} \frac{\partial}{\partial r_{\beta}(0)}=\frac{\partial}{\partial r_{\alpha}(0)}-\sum_{\beta} \varepsilon_{\alpha \beta} \frac{\partial}{\partial r_{\beta}(0)} .
$$

The Laplace operator thus becomes (up to first order in the strain)

$$
\begin{aligned}
\Delta_{r}(\varepsilon) & =\sum_{\alpha}\left(\frac{\partial}{\partial r_{\alpha}(\varepsilon)}\right)^{2} \\
& =\sum_{\alpha}\left(\frac{\partial}{\partial r_{\alpha}(0)}\right)^{2}-2 \sum_{\alpha, \beta} \varepsilon_{\alpha \beta} \frac{\partial}{\partial r_{\alpha}(0)} \frac{\partial}{\partial r_{\beta}(0)} .
\end{aligned}
$$

Hence, the strain derivative of the Laplace operator is

$$
\frac{\partial}{\partial \varepsilon_{\lambda \mu}} \Delta_{r}=-2 \frac{\partial}{\partial r_{\lambda}} \frac{\partial}{\partial r_{\mu}}
$$

and the evaluation of which requires the Hessian of the orbitals.

The strain derivative of the non-relativistic kinetic energy is an inner product of Kohn-Sham orbitals, so that we eventually obtain

$$
\frac{\partial T_{\mathrm{s}}}{\partial \varepsilon_{\lambda \mu}}=\delta_{\lambda \mu} T_{\mathrm{s}}+2 \sum_{i} f_{i}\left\langle\frac{\partial \psi_{i}}{\partial \varepsilon_{\lambda \mu}}\left|\hat{t}_{\mathrm{s}}\right| \psi_{i}\right\rangle+\sum_{i} f_{i}\left\langle\psi_{i}\left|\frac{\partial}{\partial r_{\lambda}} \frac{\partial}{\partial r_{\mu}}\right| \psi_{i}\right\rangle .
$$

\subsubsection{Scalar relativistic kinetic energy}

In FHI-aims, relativity is taken into account in a scalar relativistic approximation. Specifically, the kinetic energy operator is modified according to the "atomic ZORA" (zeroth order regular approximation with on-site free-atom potentials) [16] to approximately capture the relativistic effects. In this approximation, the kinetic energy has the following form

$$
T_{\mathrm{s}}^{\mathrm{rel}}=\frac{1}{2} \sum_{i j k, J K} f_{i} c_{i j} c_{i k}\left[\left\langle\varphi_{j}\left|\hat{t}_{\mathrm{at} . Z O R A}\right| \varphi_{k}\right\rangle+\left\langle\varphi_{k}\left|\hat{t}_{\mathrm{at} . Z O R A}\right| \varphi_{j}\right\rangle\right]
$$

with the corresponding operator

$$
\hat{t}_{\text {at.ZORA }}\left|\varphi_{j}\right\rangle=-\nabla \cdot \frac{c^{2}}{2 c^{2}-v_{\text {free }}^{J}} \nabla\left|\varphi_{j}\right\rangle .
$$

Here, $v_{\text {free }}^{J}$ is the on-site free-atom potential of atom $J$ with the associated basis function $j$.

Given that the derivative of the full expression $\hat{t}_{\text {at.ZORA }}\left|\varphi_{j}\right\rangle$ with respect to atomic coordinates is known in this formalism [16], we obtain the strain derivative

$$
\begin{aligned}
\frac{\partial T_{\mathrm{s}}^{\mathrm{rel}}}{\partial \varepsilon_{\lambda \mu}}=\delta_{\lambda \mu} T_{\mathrm{s}}^{\mathrm{rel}}+2 \sum_{i j k, J K} f_{i} c_{i j} c_{i k} & \frac{1}{2}\left[\left\langle\frac{\partial \varphi_{j}}{\partial \varepsilon_{\lambda \mu}}\left|\hat{t}_{\mathrm{at} . Z O R A}\right| \varphi_{k}\right\rangle\right. \\
& \left.+\left\langle\varphi_{k}\right| \frac{\partial}{\partial \varepsilon_{\lambda \mu}}\left(\hat{t}_{\mathrm{at} . Z O R A}\left|\varphi_{j}\right\rangle\right)\right],
\end{aligned}
$$

that includes a Jacobi term. The last derivative can be expressed in real space as

$$
\frac{\partial}{\partial \varepsilon_{\lambda \mu}}\left(\hat{t}_{\text {at.ZORA }} \varphi_{j}\left(\boldsymbol{r}-\boldsymbol{R}_{J}\right)\right)=\frac{\partial}{\partial R_{J, \lambda}}\left(\hat{t}_{\mathrm{at} . Z O R A} \varphi_{j}\left(\boldsymbol{r}-\boldsymbol{R}_{J}\right)\right)\left(R_{J, \mu}-r_{\mu}\right) .
$$

\subsection{Electrostatics}

The electrostatic energy is described by three terms: of electron-electron interaction (Hartree term) $E_{\mathrm{H}}$, the electronnuclei interaction $E_{\text {ext }}$, and the nuclei-nuclei interaction $E_{\text {nuc }}$. These three terms cannot be treated separately in a numerical approach given that - individually $-E_{\mathrm{H}}$ and $E_{\text {nuc }}$ diverge under periodic boundary conditions even per unit cell. This problem can be avoided by treating the electrostatic contributions from electrons and nuclei together by defining a total electrostatic energy, $E_{\mathrm{es}}=E_{\mathrm{H}}+E_{\mathrm{ext}}+E_{\text {nuc }}[16]$. Then, only energy contributions arising from electrostatically neutral subsystems are handled, leading to numerically much more readily converging terms.

To achieve a rapid and accurate description of the electrostatic interactions, FHI-aims follows a recipe that was proposed by Delley [21] and that has its roots in a strategy developed by Becke et al. [22]. In this formalism, the electron density $n(\boldsymbol{r})$ is partitioned into contributions $n_{I}\left(\boldsymbol{r}-\boldsymbol{R}_{I}\right)$ associated with the individual atoms $I$

$$
n_{I}\left(\boldsymbol{r}-\boldsymbol{R}_{I}\right)=\int d \boldsymbol{r} p_{I}(\boldsymbol{r}) n(\boldsymbol{r})
$$

by means of the partition function $p_{I}(\boldsymbol{r})$ defined as

$$
p_{I}(\boldsymbol{r})=\frac{g_{I}(\boldsymbol{r})}{\sum_{J} g_{J}(\boldsymbol{r})},
$$

with $g_{I}(\boldsymbol{r})$ an in principle arbitrary function that is strongly peaked at atom $I$. See Sec. 5.4 for a more in-depth discussion of the partition function and functional forms of $g_{I}$ used in FHI-aims.

The Hartree potential $v_{\mathrm{H}}(\boldsymbol{r})$ is

$$
v_{\mathrm{H}}(\boldsymbol{r})=\sum_{I} v_{\mathrm{H}}^{I}\left(\boldsymbol{r}-\boldsymbol{R}_{I}\right)=\sum_{I} \int_{V} d \tilde{\boldsymbol{r}} \frac{n_{I}\left(\tilde{\boldsymbol{r}}-\boldsymbol{R}_{I}\right)}{|\boldsymbol{r}-\tilde{\boldsymbol{r}}|}
$$




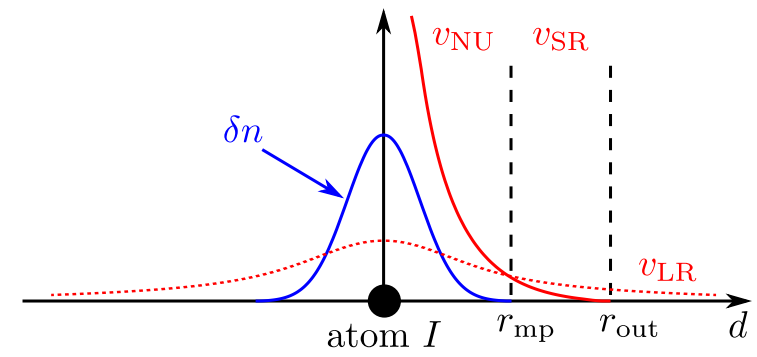

Figure 2: One dimensional sketch of the different contributions and used radii for the calculation of the electrostatic potential in FHI-aims. The abscissa shows the distance $d$ to atom $I$ and the partitioned difference density $\delta n$ of atom $I$ is depicted (blue). For distances less than $r_{\mathrm{mp}}$ where $\delta n$ is non-zero the potential $v_{\mathrm{NU}}$ (red, solid) is obtained by explicitly solving Poisson's equation. Outside the density, the potential is determined by the Ewald short range term $v_{\mathrm{SR}}$ (red, solid) which extends up to $r_{\text {out }}$. Any long range tails of the potential are attributed by the Ewald long range term $v_{\mathrm{LR}}$ (red, dotted).

and the external potential, here created by the nuclear charges $Z_{I}$, is

$$
v_{\mathrm{ext}}(\boldsymbol{r})=\sum_{I} v_{\mathrm{N}}^{I}\left(\boldsymbol{r}-\boldsymbol{R}_{I}\right)=\sum_{I} \frac{Z_{I}}{\left|\boldsymbol{r}-\boldsymbol{R}_{I}\right|} .
$$

Together, these two potentials define the total electrostatic potential, $v_{\mathrm{es}}(\boldsymbol{r})=v_{\mathrm{H}}(\boldsymbol{r})+v_{\mathrm{ext}}(\boldsymbol{r})$. Accordingly, the total electrostatic energy, which includes the electron-electron, electronnuclear, and nuclear-nuclear interaction, becomes [16]

$$
E_{\mathrm{es}}=\frac{1}{2} \int_{V} d \boldsymbol{r} n(\boldsymbol{r}) v_{\mathrm{es}}(\boldsymbol{r})+\frac{1}{2} \sum_{I} Z_{I}\left[v_{\mathrm{H}}^{I}(0)+\sum_{J \neq I} v_{\mathrm{es}}^{J}\left(\boldsymbol{R}_{J}-\boldsymbol{R}_{I}\right)\right] .
$$

In practice, the total electrostatic potential is calculated in two steps: First, the electron density associated to the superposition of free atoms $n_{\text {free }}^{I}\left(\boldsymbol{r}-\boldsymbol{R}_{I}\right)$ is used to define the difference density

$$
\delta n(\boldsymbol{r})=n(\boldsymbol{r})-\sum_{I} n_{\text {free }}^{I}\left(\boldsymbol{r}-\boldsymbol{R}_{I}\right) .
$$

with respect to the total density $n(\boldsymbol{r})$ (Eq. (7)). Second, this difference density $\delta n(\boldsymbol{r})$ is partitioned according to Eq. 47 and expanded in a multipole expansion $\delta n_{\mathrm{MP}}(\boldsymbol{r})$ (see Eq. (32) in Ref. [16]). This expansion yields an approximate description of the electron density $n(\boldsymbol{r})$, the so-called multipole density

$$
n_{\mathrm{MP}}(\boldsymbol{r})=\sum_{I} n_{\text {free }}^{I}\left(\boldsymbol{r}-\boldsymbol{R}_{I}\right)+\delta n_{\mathrm{MP}}(\boldsymbol{r})
$$

which is used for the construction of the total electrostatic potential $v_{\mathrm{es}}(\boldsymbol{r})$.

In periodic boundary conditions, the zero level of the electrostatic potential is not well defined [16]. To achieve consistency across systems, the zero of the electrostatic potential is manually set to the spatial average of the potential $\delta v_{\mathrm{es}}(\boldsymbol{r})$ pertaining to the difference density $\delta n_{\mathrm{MP}}(\boldsymbol{r})$

$$
v_{\mathrm{avg}}=\frac{1}{V} \int_{V} d \boldsymbol{r} \delta v_{\mathrm{es}}(\boldsymbol{r})
$$

Accordingly, the total electrostatic potential consists of three terms

$$
v_{\mathrm{es}}(\boldsymbol{r})=\sum_{I} v_{\text {free }}^{I}\left(\boldsymbol{r}-\boldsymbol{R}_{I}\right)+\delta v_{\mathrm{es}}(\boldsymbol{r})-v_{\mathrm{avg}} .
$$

Here, the potentials $v_{\text {free }}^{I}(\boldsymbol{r})$ of the free atoms are calculated as a cubic spline function on dense logarithmic grids, while the potential $\delta v_{\mathrm{es}}(\boldsymbol{r})$ is split up into three contributions:

$$
\delta v_{\mathrm{es}}(\boldsymbol{r})=\sum_{I}\left[v_{\mathrm{NU}}^{I}\left(\boldsymbol{r}-\boldsymbol{R}_{I}\right)+v_{\mathrm{SR}}^{I}\left(\boldsymbol{r}-\boldsymbol{R}_{I}\right)+v_{\mathrm{LR}}^{I}\left(\boldsymbol{r}-\boldsymbol{R}_{I}\right)\right] .
$$

This potential is calculated with the help of the Ewald summation for multipole charges [18]. The first term is the numerical potential $v_{\mathrm{NU}}^{I}\left(\boldsymbol{r}-\boldsymbol{R}_{I}\right)$ which solves the electrostatic potential explicitly for short ranges $d_{I}=\left|\boldsymbol{r}-\boldsymbol{R}_{I}\right|<r_{\mathrm{mp}}$ where the partitioned difference density of atom $I$ is non-zero, see Fig. 2. This is done by the Green's function for Poisson's equation of multipoles from classical electrostatics (see Eq. (33) in Ref. [16]). Please note, that $v_{\mathrm{NU}}^{I}\left(\boldsymbol{r}-\boldsymbol{R}_{I}\right)$ depends only on positions vectors. The other terms are the Ewald short range potential [18]

$$
v_{\mathrm{SR}}^{I}\left(\boldsymbol{r}-\boldsymbol{R}_{I}\right)=\sum_{l, m} Q_{I, l m} E_{l m} F^{p(l m)}\left(\left|\boldsymbol{r}-\boldsymbol{R}_{I}\right|\right)\left(d_{I}^{x}\right)^{X_{l m}}\left(d_{I}^{y}\right)^{Y_{l m}}\left(d_{I}^{z}\right)^{Z_{l m}},
$$

which extends up to a specific distance $r_{\text {out }}$ from atom $I$, see Fig. 2, and the Ewald long range potential [18]

$$
v_{\mathrm{LR}}^{I}\left(\boldsymbol{r}-\boldsymbol{R}_{I}\right)=\frac{4 \pi}{V} \sum_{G} \frac{\exp \left(-\frac{\kappa^{2} G^{2}}{4}\right)}{G^{2}} S_{I}(\boldsymbol{G}) \exp (i \boldsymbol{G} \boldsymbol{r})
$$

with

$$
S_{I}(\boldsymbol{G})=\sum_{l, m} Q_{I, l m} G_{l m} \exp \left(-i \boldsymbol{G} \boldsymbol{R}_{I}\right) i^{D_{l m}}\left(G_{x}\right)^{A_{l m}}\left(G_{y}\right)^{B_{l m}}\left(G_{z}\right)^{C_{l m}} .
$$

Thereby, $E_{l m}, G_{l m}$, the exponents $A_{l m}, B_{l m}, C_{l m}, D_{l m}, X_{l m}, Y_{l m}$, $Z_{l m}$, and the function $F^{p(l m)}$ are expressions that only depend on the orbital $(l)$ and magnetic $(m)$ quantum numbers. The exact form of these terms is not relevant in this context but can be looked up in the publication by Delley [18]. The $Q_{I, l m}$ are the atomic multipoles and $\kappa$ is the Ewald parameter.

To reduce the respective error in the total electrostatic energy expression to quadratic order with respect to the maximal angular momentum used in the expansion [23], the first term in Eq. 51] becomes

$$
\frac{1}{2} \int_{V} d \boldsymbol{r} n(\boldsymbol{r}) v_{\mathrm{es}}(\boldsymbol{r}) \longrightarrow \int_{V} d \boldsymbol{r}\left[n(\boldsymbol{r})-\frac{1}{2} n_{\mathrm{MP}}(\boldsymbol{r})\right] v_{\mathrm{es}}(\boldsymbol{r})
$$

For a more detailed derivation, see the FHI-aims publication [16] and the references therein.

Taking the strain derivative of the total electrostatic energy (Eq. 51 and (60) results in many different terms that cannot be simplified

$$
\begin{aligned}
\frac{\partial E_{\mathrm{es}}}{\partial \varepsilon_{\lambda \mu}}= & \delta_{\lambda \mu} \int_{V} d \boldsymbol{r}\left[n(\boldsymbol{r})-\frac{1}{2} n_{\mathrm{MP}}(\boldsymbol{r})\right] v_{\mathrm{es}}(\boldsymbol{r}) \\
& +\int_{V} d \boldsymbol{r}\left[n(\boldsymbol{r})-\frac{1}{2} n_{\mathrm{MP}}(\boldsymbol{r})\right] \frac{\partial v_{\mathrm{es}}(\boldsymbol{r})}{\partial \varepsilon_{\lambda \mu}} \\
& +\int_{V} d \boldsymbol{r}\left[\frac{\partial n(\boldsymbol{r})}{\partial \varepsilon_{\lambda \mu}}-\frac{1}{2} \frac{\partial n_{\mathrm{MP}}(\boldsymbol{r})}{\partial \varepsilon_{\lambda \mu}}\right] v_{\mathrm{es}}(\boldsymbol{r}) \\
& +\frac{1}{2} \sum_{I, J \neq I} Z_{I} \frac{\partial v_{\mathrm{es}}^{J}\left(\boldsymbol{R}_{I}\right)}{\partial \varepsilon_{\lambda \mu}} .
\end{aligned}
$$


The term $\int_{V} d \boldsymbol{r} \frac{\partial n(\boldsymbol{r})}{\partial \varepsilon_{\lambda \mu}} v_{\mathrm{es}}(\boldsymbol{r})$ can be again rewritten as an inner product of Kohn-Sham orbitals, namely $2 \sum_{i} f_{i}\left\langle\frac{\partial \psi_{i}}{\partial \varepsilon_{\lambda \mu}}\left|\hat{v}_{\mathrm{es}}\right| \psi_{i}\right\rangle$. The strain derivative of $n_{\mathrm{MP}}(\boldsymbol{r})$ can be evaluated by using the partitioning of the multipole density into single atom contributions $n_{\mathrm{MP}}^{I}\left(\boldsymbol{r}-\boldsymbol{R}_{I}\right)(\mathrm{Eq} .47)$

$$
\begin{aligned}
\frac{\partial n_{\mathrm{MP}}(\boldsymbol{r})}{\partial \varepsilon_{\lambda \mu}} & =\sum_{I} \frac{\partial n_{\mathrm{MP}}^{I}\left(\boldsymbol{r}-\boldsymbol{R}_{I}\right)}{\partial \varepsilon_{\lambda \mu}} \\
& =\sum_{I} \frac{\partial n_{\mathrm{MP}}^{I}\left(\boldsymbol{r}-\boldsymbol{R}_{I}\right)}{\partial r_{\lambda}}\left(r_{\mu}-R_{I, \mu}\right) .
\end{aligned}
$$

The strain derivative of the on-site term (the atom interacts with its own electrons) $Z_{I} v_{\mathrm{H}}^{I}(0)$ that appears in Eq. 51] vanishes, since the atom and its electron density transform equally under a strain deformation. A rigorous mathematical proof can be found in Appendix A.1.

In Eq. (61), the strain derivative of the total electrostatic potential $v_{\mathrm{es}}(\boldsymbol{r})$ requires some more work. We have to calculate the individual strain derivatives of Eq. (54), 55), and (56). The terms $v_{\text {free }}^{I}\left(\boldsymbol{r}-\boldsymbol{R}_{I}\right), v_{\mathrm{NU}}^{I}\left(\boldsymbol{r}-\boldsymbol{R}_{I}\right)$, and $v_{\mathrm{SR}}^{I}\left(\boldsymbol{r}-\boldsymbol{R}_{I}\right)$ depend only position vectors, thus, their derivatives are simply

$$
\begin{aligned}
& \frac{\partial v_{\mathrm{free}}^{I}\left(\boldsymbol{r}-\boldsymbol{R}_{I}\right)}{\partial \varepsilon_{\lambda \mu}}=\frac{\partial v_{\mathrm{free}}^{I}\left(\boldsymbol{r}-\boldsymbol{R}_{I}\right)}{\partial R_{I, \lambda}}\left(R_{I, \mu}-r_{\mu}\right) \\
& \frac{\partial v_{\mathrm{NU}}^{I}\left(\boldsymbol{r}-\boldsymbol{R}_{I}\right)}{\partial \varepsilon_{\lambda \mu}}=\frac{\partial v_{\mathrm{NU}}^{I}\left(\boldsymbol{r}-\boldsymbol{R}_{I}\right)}{\partial R_{I, \lambda}}\left(R_{I, \mu}-r_{\mu}\right) \\
& \frac{\partial v_{\mathrm{SR}}^{I}\left(\boldsymbol{r}-\boldsymbol{R}_{I}\right)}{\partial \varepsilon_{\lambda \mu}}=\frac{\partial v_{\mathrm{SR}}^{I}\left(\boldsymbol{r}-\boldsymbol{R}_{I}\right)}{\partial R_{I, \lambda}}\left(R_{I, \mu}-r_{\mu}\right) .
\end{aligned}
$$

The most complicated term is $v_{\mathrm{LR}}^{I}\left(\boldsymbol{r}-\boldsymbol{R}_{I}\right)$ because it depends on volume, position and $\boldsymbol{G}$-vectors. Using Eq. (13) yields

$$
\begin{aligned}
\frac{\partial v_{\mathrm{LR}}^{I}\left(\boldsymbol{r}-\boldsymbol{R}_{I}\right)}{\partial \varepsilon_{\lambda \mu}}= & \frac{v_{\mathrm{LR}}^{I}\left(\boldsymbol{r}-\boldsymbol{R}_{I}\right)}{\partial r_{\lambda}} r_{\mu}+\frac{\partial v_{\mathrm{LR}}^{I}\left(\boldsymbol{r}-\boldsymbol{R}_{I}\right)}{\partial R_{I_{\lambda}}} R_{I, \mu} \\
& -\frac{\partial v_{\mathrm{LR}}^{I}\left(\boldsymbol{r}-\boldsymbol{R}_{I}\right)}{\partial G_{\mu}} G_{\lambda}+\frac{\partial v_{\mathrm{LR}}^{I}\left(\boldsymbol{r}-\boldsymbol{R}_{I}\right)}{\partial V} \delta_{\lambda \mu} V .
\end{aligned}
$$

Evaluating the individual derivatives results in the final expression

$$
\begin{aligned}
\frac{\partial v_{\mathrm{LR}}^{I}\left(\boldsymbol{r}-\boldsymbol{R}_{I}\right)}{\partial \varepsilon_{\lambda \mu}}=\frac{4 \pi}{V} & \sum_{G} \frac{\exp \left(-\frac{\kappa^{2} G^{2}}{4}\right)}{G^{2}} \exp (i \boldsymbol{G r}) \\
& \times\left[S_{I}(\boldsymbol{G}) \Lambda_{\lambda \mu}(\boldsymbol{G})+G_{\lambda} \Gamma_{I, \mu}(\boldsymbol{G})\right]
\end{aligned}
$$

with

$$
\begin{aligned}
& \Lambda_{\lambda \mu}(\boldsymbol{G})=-\delta_{\lambda \mu}+\left(\frac{2}{G^{2}}+\frac{\kappa^{2}}{2}\right) G_{\lambda} G_{\mu} \\
& \Gamma_{I, \mu}(\boldsymbol{G})=\sum_{l, m} Q_{I, l m} G_{l m} \exp \left(-i \boldsymbol{G} \boldsymbol{R}_{I}\right) i^{D_{l m}} \gamma_{\mu}^{l m}(\boldsymbol{G}) \\
& \gamma_{\mu}^{l m}(\boldsymbol{G})=\frac{\partial}{\partial G_{\mu}}\left[\left(G_{x}\right)^{A_{l m}}\left(G_{y}\right)^{B_{l m}}\left(G_{z}\right)^{C_{l m}}\right] .
\end{aligned}
$$

For an explicit derivation of the individual derivatives of $v_{\mathrm{LR}}^{I}(\boldsymbol{r}-$ $\boldsymbol{R}_{I}$ ), see Appendix A.2. The strain derivative of the average potential $v_{\text {avg }}$ (Eq. (54)) introduces nothing new and only already calculated derivatives appear (the ones of $\delta v_{\mathrm{es}}(\boldsymbol{r})$ ). Please note that no Jacobian term appears here due to the prefactor $1 / V$ of $v_{\text {avg. }}$.

\subsection{Exchange-correlation energy}

\subsection{1. $L D A$}

For exchange-correlation functionals at the level of the localdensity approximation (LDA) [24-26] the exchange-correlation energy per particle $\epsilon_{\mathrm{xc}}$ is a smooth function of the electronic density $n(\boldsymbol{r})$, so that the exchange-correlation energy has the following form

$$
E_{\mathrm{xc}}^{\mathrm{LDA}}=\int_{V} d \boldsymbol{r} n(\boldsymbol{r}) \epsilon_{\mathrm{xc}}^{\mathrm{LDA}}(n(\boldsymbol{r})) .
$$

Its strain derivative can be derived using the chain rule and the definition of the exchange-correlation potential $v_{\mathrm{xc}}^{\mathrm{LDA}}(n)$ for LDA functionals:

$$
\begin{aligned}
\frac{\partial E_{\mathrm{xc}}^{\mathrm{LDA}}}{\partial \varepsilon_{\lambda \mu}} & =\delta_{\lambda \mu} E_{\mathrm{xc}}^{\mathrm{LDA}}+\int_{V} d \boldsymbol{r} \frac{\partial n(\boldsymbol{r})}{\partial \varepsilon_{\lambda \mu}}\left[\epsilon_{\mathrm{xc}}^{\mathrm{LDA}}(n)+n(\boldsymbol{r}) \frac{\partial \epsilon_{\mathrm{xc}}^{\mathrm{LDA}}(n)}{\partial n(\boldsymbol{r})}\right] \\
& =\delta_{\lambda \mu} E_{\mathrm{xc}}^{\mathrm{LDA}}+2 \sum_{i} f_{i}\left\langle\frac{\partial \psi_{i}}{\partial \varepsilon_{\lambda \mu}}\left|\hat{v}_{\mathrm{xc}}^{\mathrm{LDA}}(n)\right| \psi_{i}\right\rangle .
\end{aligned}
$$

We obtain again two terms because we integrate over the unit cell volume. The integral over the strain derivative of the electronic density was rewritten as a sum of inner products between the Kohn-Sham orbitals and their respective strain derivatives according to Eq. (7).

\subsection{2. $G G A$}

In the generalized gradient approximation (GGA), e.g., the PBE [27] and BLYP [28, 29] functional, the exchangecorrelation energy has the same overall form as in the LDA case

$$
E_{\mathrm{xc}}^{\mathrm{GGA}}=\int_{V} d \boldsymbol{r} n(\boldsymbol{r}) \epsilon_{\mathrm{xc}}^{\mathrm{GGA}}(n(\boldsymbol{r}), \nabla n(\boldsymbol{r})),
$$

but in addition, the exchange-correlation energy per particle $\epsilon_{\mathrm{xc}}^{\mathrm{GGA}}(n(\boldsymbol{r}), \nabla n(\boldsymbol{r}))$ now depends on the density gradient $\nabla n$, which gives rise to an additional term in the strain derivative of $\epsilon_{\mathrm{xc}}^{\mathrm{GGA}}$ :

$$
\begin{aligned}
\frac{\partial E_{\mathrm{xc}}^{\mathrm{GGA}}}{\partial \varepsilon_{\lambda \mu}}= & \delta_{\lambda \mu} E_{\mathrm{xc}}^{\mathrm{GGA}}+\int_{V} d \boldsymbol{r} \frac{\partial n(\boldsymbol{r})}{\partial \varepsilon_{\lambda \mu}}\left[\epsilon_{\mathrm{xc}}^{\mathrm{GGA}}+n(\boldsymbol{r}) \frac{\partial \epsilon_{\mathrm{xc}}^{\mathrm{GGA}}}{\partial n(\boldsymbol{r})}\right] \\
& +2 \int_{V} d \boldsymbol{r} n(\boldsymbol{r})\left((\nabla n) \cdot \frac{\partial \nabla n}{\partial \varepsilon_{\lambda \mu}}\right) \frac{\partial \epsilon_{\mathrm{xc}}^{\mathrm{GGA}}}{\partial|\nabla n|^{2}}
\end{aligned}
$$

The term in parentheses in the last line corresponds to a scalar product of vectors. We define a local exchange-correlation potential for GGA which includes only the partial derivative with 
respect to $n, \hat{v}_{\mathrm{xc}, \mathrm{loc}}^{\mathrm{GGA}}=\epsilon_{\mathrm{xc}}^{\mathrm{GGA}}+n \frac{\partial \epsilon_{\mathrm{xc}}^{\mathrm{GGA}}}{\partial n}$, and the last line can be expanded by using Eq. (7) and the product rule for derivatives

$$
\begin{aligned}
\frac{\partial E_{\mathrm{xc}}^{\mathrm{GGA}}}{\partial \varepsilon_{\lambda \mu}}= & \delta_{\lambda \mu} E_{\mathrm{xc}}^{\mathrm{GGA}}+2 \sum_{i} f_{i}\left\langle\frac{\partial \psi_{i}}{\partial \varepsilon_{\lambda \mu}}\left|\hat{v}_{\mathrm{xc}, \mathrm{loc}}^{\mathrm{GGA}}(n)\right| \psi_{i}\right\rangle \\
& +4 \sum_{i} f_{i} \int_{V} d \boldsymbol{r}(\nabla n) \cdot\left[\frac{\partial \psi_{i}}{\partial \varepsilon_{\lambda \mu}} \nabla \psi_{i}+\frac{\partial \nabla \psi_{i}}{\partial \varepsilon_{\lambda \mu}} \psi_{i}\right] .
\end{aligned}
$$

\subsubsection{Hybrid functionals}

In hybrid exchange-correlation functionals, semi-local GGA exchange-correlation is mixed with the non-local Hartree-Fock (HF) like exchange [30, 31], the so-called exact exchange and various hybrid functionals exist in the literature. In the simplest one parameter case $2^{2}$, a fraction $\alpha$ of the GGA exchange is replaced by $\mathrm{HF}$ exchange and the respective exchange-correlation energy takes the following form

$$
E_{\mathrm{xc}}^{\mathrm{hyb}}=E_{\mathrm{xc}}^{\mathrm{GGA}}+\alpha\left(E_{\mathrm{x}}^{\mathrm{HF}}-E_{\mathrm{x}}^{\mathrm{GGA}}\right) .
$$

The corner cases with $\alpha$ equals 1 or 0 correspond to a pure Hartree-Fock or pure GGA calculation, respectively. In the case of the PBE0 functional [32], $\alpha=1 / 4$ and the GGA-PBE exchange and correlation are used in the definition (76). Furthermore, it is possible to introduce a range separation: For instance, the Coulomb interaction of the HF exchange is screened in the case of the HSE family of functionals [33- -35$]$ and the exchange-correlation energy is of the form

$$
E_{\mathrm{xc}}^{\mathrm{hyb}}=E_{\mathrm{xc}}^{\mathrm{PBE}}+\alpha\left(E_{\mathrm{x}}^{\mathrm{HF}, \mathrm{SR}}(\omega)-E_{\mathrm{x}}^{\mathrm{PBE}, \mathrm{SR}}(\omega)\right),
$$

where $\omega$ is the screening parameter (the higher $\omega$, the stronger the screening). $\omega=0$ corresponds to the PBE0 functional (with $\alpha=1 / 4)$ and $\omega \rightarrow \infty$ to the PBE function. The modification of the exchange-correlation energy implicates a change of the exchange-correlation potential in Eq. (8), too, such that

$$
\hat{v}_{\mathrm{xc}}^{\mathrm{hyb}}=\hat{v}_{\mathrm{xc}}^{\mathrm{GGA}}+\alpha\left(\hat{\Sigma}_{\mathrm{x}}(\omega)-\hat{v}_{\mathrm{x}}^{\mathrm{GGA}}(\omega)\right) .
$$

with $\hat{\Sigma}_{\mathrm{x}}$ the exact-exchange potential.

Here, we only concisely describe how the exact-exchange energy is actually calculated following the notation of paper [36], in which more detailed derivations can be found. For a given basis set expansion as defined in Eq. (9), the exact-exchange energy is defined as

$$
E_{\mathrm{x}}^{\mathrm{HF}}=-\frac{1}{2} \sum_{i j k l} D_{j k} D_{i l}(i j \mid k l),
$$

where the indices $i, j, k, l$ run over all basis functions. Here, $D_{j k}=\sum_{m} f_{m} c_{m j} c_{m k}$ is the one-particle density matrix for the occupation numbers $f_{m}$ and the expansions coefficients $c_{m j}$ introduced before. The expression $(i j \mid k l)$ represents the four-center two-electron Coulomb integral

$$
(i j \mid k l)=\iint d \boldsymbol{r} d \tilde{\boldsymbol{r}} \varphi_{i}(\boldsymbol{r}) \varphi_{j}(\boldsymbol{r}) v(|\boldsymbol{r}-\tilde{\boldsymbol{r}}|) \varphi_{k}(\tilde{\boldsymbol{r}}) \varphi_{l}(\tilde{\boldsymbol{r}})
$$

\footnotetext{
${ }^{2}$ Our derivations are easily extended to functionals with more than one parameter since the individual ingredients are solely the exchange and/or correlation of LDA, GGA, or HF which are all covered in this paper.
}

with the Coulomb kernel $v(|\boldsymbol{r}|)$. Please note, that no complex conjugate quantities appear since the basis functions are chosen to be real valued in FHI-aims. For HF and PBE0 the Coulomb kernel is simply the Coulomb potential $1 /|\boldsymbol{r}|$, for HSE06 $v(|\boldsymbol{r}|)=$ $\operatorname{erfc}(\omega|\boldsymbol{r}|) /|\boldsymbol{r}|$ such that only the short-range part of the Coulomb potential is included.

With help of the resolution of identity (RI) [37], one can expand the product $\varphi_{i}(\boldsymbol{r}) \varphi_{j}(\boldsymbol{r})$ of two atomic orbitals (centered at atom $I$ and $J$, respectively) in an auxiliary, product basis set $P_{\mathrm{m}}(\boldsymbol{r})$

$$
\varphi_{i}(\boldsymbol{r}) \varphi_{j}(\boldsymbol{r}) \approx \sum_{\mathrm{m}} C_{i j}^{\mathfrak{m}} P_{\mathfrak{m}}(\boldsymbol{r})
$$

Accordingly, the exact-exchange energy can be rewritten as

$$
E_{\mathrm{x}}^{\mathrm{HF}}=-\frac{1}{2} \sum_{\substack{i j k l \\ \mathrm{~m} \mathfrak{n}}} D_{j k} D_{i l}\left[C_{i j}^{\mathrm{m}} V_{\mathrm{mm}} C_{k l}^{\mathfrak{n}}\right]
$$

with the Coulomb matrix $V_{\mathfrak{m}}=(\mathrm{m} \mid \mathrm{n})$. In the so-called RI-V formalism, the error in the four-center Coulomb integrals that stems from the product basis expansion is minimized [38] by choosing the expansion coefficients according to

$$
C_{i j}^{\mathrm{m}}=\sum_{\mathfrak{n}}(i j \mid \mathfrak{n}) V_{\mathfrak{m}}^{-1}
$$

Within negligible losses in the accuracy, the computational effort can be reduced drastically by using a locally restricted expansion in the so-called RI-LVL formalism [39, 40]

$$
\varphi_{i}(\boldsymbol{r}) \varphi_{j}(\boldsymbol{r}) \approx \sum_{\mathfrak{m} \in \mathcal{P}(I J)} C_{i j}^{\mathfrak{m}} P_{\mathfrak{m}}(\boldsymbol{r})
$$

with $\mathcal{P}(I J)=\mathcal{P}(I) \cup \mathcal{P}(J)$. The set of all auxiliary basis functions that are centered at atom $I$ is denoted with $\mathcal{P}(I)$. Consequently, the expansion coefficients become

$$
C_{i j}^{\mathfrak{m}}= \begin{cases}\sum_{\mathfrak{n} \in \mathcal{P}(I J)}(i j \mid \mathfrak{n}) L_{\mathfrak{n} \mathfrak{m}}^{I J} & \text { for } \mathrm{m} \in \mathcal{P}(I J) \\ 0 & \text { else }\end{cases}
$$

with $L^{I J}=\left(V^{I J}\right)^{-1}$ the inverse of the Coulomb matrix including only auxiliary basis functions centered at atom $I$ or $J$.

The strain derivative of the exact-exchange energy in the LVL-scheme is

$$
\frac{\partial E_{\mathrm{x}}^{\mathrm{HF}}}{\partial \varepsilon_{\lambda \mu}}=-\frac{1}{2} \sum_{\substack{i j k l \\ \mathfrak{m}, \mathrm{n} \in \mathcal{P}(I J)}} D_{j k} D_{i l}\left[C_{i j}^{\mathfrak{m}} \frac{\partial V_{\mathrm{mm}}}{\partial \varepsilon_{\lambda \mu}} C_{k l}^{\mathfrak{n}}+2 \frac{\partial C_{i j}^{\mathfrak{m}}}{\partial \varepsilon_{\lambda \mu}} V_{\mathfrak{m} \mathfrak{1}} C_{k l}^{\mathfrak{n}}\right] .
$$

In this case, no Jacobian terms arise, since the integration domains of the Coulomb integrals range over the whole space.

In the following, we will use the notation $i(I)$ to indicate that the basis function $i$ is centered on atom $I$. The strain derivative of the Coulomb matrix then becomes

$$
\begin{aligned}
\frac{\partial V_{\mathfrak{m}(I) \mathfrak{n}(J)}}{\partial \varepsilon_{\lambda \mu}} & =\frac{\partial V_{\mathfrak{m}(I) \mathfrak{n}(J)}}{\partial R_{I, \lambda}} R_{I, \mu}+\frac{\partial V_{\mathfrak{m}(I) \mathfrak{n}(J)}}{\partial R_{J, \lambda}} R_{J, \mu} \\
& =\frac{\partial V_{\mathfrak{m}(I) \mathfrak{n}(J)}}{\partial R_{I, \lambda}}\left(R_{I, \mu}-R_{J, \mu}\right) .
\end{aligned}
$$


For the expansion coefficients, we get an analogous result

$$
\begin{aligned}
\frac{\partial C_{i(I) j(J)}^{\mathfrak{m}(\mathfrak{M})}}{\partial \varepsilon_{\lambda \mu}} & =\frac{\partial C_{i(I) j(J)}^{\mathfrak{m}(\mathfrak{M})}}{\partial R_{I, \lambda}} R_{I, \mu}+\frac{\partial C_{i(I) j(J)}^{\mathfrak{m}(\mathfrak{M})}}{\partial R_{J, \lambda}} R_{J, \mu} \\
& =\frac{\partial C_{i(I) j(J)}^{\mathfrak{m}(\mathfrak{M})}}{\partial R_{I, \lambda}}\left(R_{I, \mu}-R_{J, \mu}\right),
\end{aligned}
$$

where $\mathfrak{M}$ is either atom $I$ or $J$. A detailed proof of Eq. 87] and (88) can be found in Appendix B.

Using the relations derived above and in Sec. 3.5.2, the strain derivative of the hybrid exchange-correlation functional is

$$
\begin{aligned}
& \frac{\partial E_{\mathrm{xc}}^{\mathrm{hyb}}}{\partial \varepsilon_{\lambda \mu}}= \frac{\partial E_{\mathrm{xc}}^{\mathrm{GGA}}}{\partial \varepsilon_{\lambda \mu}}-\alpha \frac{\partial E_{\mathrm{x}}^{\mathrm{GGA}}}{\partial \varepsilon_{\lambda \mu}} \\
&-\frac{\alpha}{2} \sum_{\substack{i j k l \\
\mathfrak{m}, \mathrm{n} \in \mathcal{P}(I J)}} D_{j k} D_{i l}\left(R_{I, \mu}-R_{J, \mu}\right) \\
& \\
& \quad \times\left[C_{i j}^{\mathfrak{m}} \frac{\partial V_{\mathrm{mu}}}{\partial R_{I, \lambda}} C_{k l}^{\mathfrak{n}}+2 \frac{\partial C_{i j}^{\mathrm{m}}}{\partial R_{I, \lambda}} V_{\mathrm{mm}} C_{k l}^{\mathfrak{n}}\right],
\end{aligned}
$$

where the current center of the basis functions $i, j, k, l$ and of the auxiliary basis functions $\mathrm{m}, \boldsymbol{n}$ is atom $I$ or $J$.

\subsubsection{Van der Waals correction}

Commonly used exchange-correlation functionals such as LDA, GGA, and hybrids lack the long range tail of van der Waals interactions. In order to incorporate these long range contributions in our DFT calculation, we employ the correction scheme proposed by Tkatchenko and Scheffler [17]. Like in many other methods [41-43], a pairwise interatomic correction term $\left(E_{\mathrm{vdW}}\right)$ is added to the exchange-correlation energy of the GGA functionals such that

$$
E_{\mathrm{xc}}^{\mathrm{GGA}} \rightarrow E_{\mathrm{xc}}^{\mathrm{GGA}}+E_{\mathrm{vdW}}
$$

The term for the long range tail of van der Waals interactions is given by

$$
E_{\mathrm{vdW}}=-\frac{1}{2} \sum_{I, J} f_{\mathrm{damp}}\left(R_{I J}\right) \frac{C_{6 I J}}{R_{I J}^{6}}
$$

with $R_{I J}$ the distance between atom $I$ and $J$ and the corresponding $C_{6 I J}$ coefficient which is determined from the ground-state electron density. The onset distance of the vdW correction is controlled by the damping function

$$
f_{\text {damp }}\left(R_{I J}\right)=\frac{1}{1+\exp \left[-d\left(\frac{R_{I J}}{s_{R} R_{I J}^{\text {eff }}}-1\right)\right]},
$$

in which $d$ and $s_{R}$ are tabulated parameters. $R_{I J}^{\mathrm{eff}}=R_{I}^{\mathrm{eff}}+R_{J}^{\mathrm{eff}}$ is the sum of the effective vdW radii, which are determined from the ground-state electron density [17]. With the help of Eq. (15), the strain derivative of the vdW correction is

$$
\frac{\partial E_{\mathrm{vdW}}}{\partial \varepsilon_{\lambda \mu}}=-\frac{1}{2} \sum_{I, J} \frac{C_{6 I J}}{R_{I J}^{6}}\left[\frac{\partial f_{\mathrm{damp}}\left(R_{I J}\right)}{\partial R_{I J, \lambda}}-6 \frac{R_{I J, \lambda}}{R_{I J}^{2}}\right] R_{I J, \mu}
$$

Here, we neglected the change of the $C_{6}$ coefficients and the dampening function under strain since they seem to be negligible in practice [44].

\subsection{Summary of terms - the stress tensor}

We will now collect all contributions to the stress tensor we have derived in the previous sections. Additionally, we will replace $\epsilon_{i}$ in the first term of Eq. (37) by the Hamiltonian of Eq. (8) such that some terms cancel. Hence, the stress tensor is

$$
\sigma_{\lambda \mu}=\sigma_{\lambda \mu}^{\mathrm{norm}}+\sigma_{\lambda \mu}^{\mathrm{kin}}+\sigma_{\lambda \mu}^{\mathrm{el}, \mathrm{n}}+\sigma_{\lambda \mu}^{\mathrm{el}, \mathrm{e}}+\sigma_{\lambda \mu}^{\mathrm{el}, \mathrm{MP}}+\sigma_{\lambda \mu}^{\mathrm{xc}}+\sigma_{\lambda \mu}^{\mathrm{vdW}}
$$

with

$$
\begin{aligned}
& \sigma_{\lambda \mu}^{\mathrm{norm}}=-\frac{2}{V} \sum_{i} f_{i}\left\langle\frac{\partial \psi_{i}}{\partial \varepsilon_{\lambda \mu}}\left|\epsilon_{i}\right| \psi_{i}\right\rangle \\
& \sigma_{\lambda \mu}^{\text {kin,non-rel }}=\frac{2}{V} \sum_{i} f_{i}\left\langle\frac{\partial \psi_{i}}{\partial \varepsilon_{\lambda \mu}}\left|\hat{t}_{\mathrm{s}}\right| \psi_{i}\right\rangle+\frac{1}{V} \sum_{i} f_{i}\left\langle\psi_{i}\left|\frac{\partial}{\partial r_{\lambda}} \frac{\partial}{\partial r_{\mu}}\right| \psi_{i}\right\rangle \\
& \sigma_{\lambda \mu}^{\mathrm{kin}, \mathrm{rel}}=\frac{1}{V} \sum_{i j k k, J K} f_{i} c_{i j} c_{i k}\left[\left\langle\frac{\partial \varphi_{j}}{\partial \varepsilon_{\lambda \mu}}\left|\hat{t}_{\mathrm{at} . Z O R A}\right| \varphi_{k}\right\rangle\right. \\
& \left.+\left\langle\varphi_{k}\right|\left(R_{J, \mu}-r_{\mu}\right) \frac{\partial}{\partial R_{J, \lambda}}\left(\hat{t}_{\text {at.ZORA }}\left|\varphi_{j}\right\rangle\right)\right] \\
& \sigma_{\lambda \mu}^{\mathrm{el}, \mathrm{n}}=\frac{1}{2 V} \sum_{I, J \neq I} Z_{I} \frac{\partial v_{\mathrm{es}}^{J}\left(\boldsymbol{R}_{I}\right)}{\partial \varepsilon_{\lambda \mu}} \\
& \sigma_{\lambda \mu}^{\mathrm{el}, \mathrm{e}}=\frac{1}{V} \sum_{i} f_{i}\left[2\left\langle\frac{\partial \psi_{i}}{\partial \varepsilon_{\lambda \mu}}\left|\hat{v}_{\mathrm{es}}\right| \psi_{i}\right\rangle+\left\langle\psi_{i}\left|\frac{\partial \hat{v}_{\mathrm{es}}(\boldsymbol{r})}{\partial \varepsilon_{\lambda \mu}}\right| \psi_{i}\right\rangle\right] \\
& \sigma_{\lambda \mu}^{\mathrm{el}, \mathrm{MP}}=-\delta_{\lambda \mu} \frac{1}{2 V} \int_{V} d \boldsymbol{r} n_{\mathrm{MP}}(\boldsymbol{r}) v_{\mathrm{es}}(\boldsymbol{r}) \\
& -\frac{1}{2 V} \sum_{I} \int_{V} d \boldsymbol{r} \frac{\partial n_{\mathrm{MP}}^{I}\left(\boldsymbol{r}-\boldsymbol{R}_{I}\right)}{\partial r_{\lambda}}\left(r_{\mu}-R_{I, \mu}\right) v_{\mathrm{es}}(\boldsymbol{r}) \\
& -\frac{1}{2 V} \int_{V} d \boldsymbol{r} n_{\mathrm{MP}}(\boldsymbol{r}) \frac{\partial v_{\mathrm{es}}(\boldsymbol{r})}{\partial \varepsilon_{\lambda \mu}} \\
& \sigma_{\lambda \mu}^{\mathrm{xc}, \mathrm{LDA}}=\delta_{\lambda \mu} \frac{1}{V}\left(E_{\mathrm{xc}}^{\mathrm{LDA}}-\sum_{i} f_{i}\left\langle\psi_{i}\left|\hat{v}_{\mathrm{xc}}^{\mathrm{LDA}}\right| \psi_{i}\right\rangle\right) \\
& +\frac{2}{V} \sum_{i} f_{i}\left\langle\frac{\partial \psi_{i}}{\partial \varepsilon_{\lambda \mu}}\left|\hat{v}_{\mathrm{xc}}^{\mathrm{LDA}}\right| \psi_{i}\right\rangle \\
& \sigma_{\lambda \mu}^{\mathrm{xc}, \mathrm{GGA}}=\delta_{\lambda \mu} \frac{1}{V}\left(E_{\mathrm{xc}}^{\mathrm{GGA}}-\sum_{i} f_{i}\left\langle\psi_{i}\left|\hat{v}_{\mathrm{xc}}^{\mathrm{GGA}}\right| \psi_{i}\right\rangle\right) \\
& +\frac{2}{V} \sum_{i} f_{i}\left\langle\frac{\partial \psi_{i}}{\partial \varepsilon_{\lambda \mu}}\left|\hat{v}_{\mathrm{xc}, \mathrm{loc}}^{\mathrm{GGA}}\right| \psi_{i}\right\rangle \\
& +\frac{4}{V} \sum_{i} f_{i} \int_{V} d \boldsymbol{r}(\nabla n) \cdot\left[\frac{\partial \psi_{i}}{\partial \varepsilon_{\lambda \mu}} \nabla \psi_{i}+\frac{\partial \nabla \psi_{i}}{\partial \varepsilon_{\lambda \mu}} \psi_{i}\right] \\
& \sigma_{\lambda \mu}^{\mathrm{xc}, \mathrm{hyb}}=\sigma_{\lambda \mu}^{\mathrm{xc}, \mathrm{GGA}}-\alpha \sigma_{\lambda \mu}^{\mathrm{x}, \mathrm{GGA}}-\delta_{\lambda \mu} \frac{2 \alpha}{V} E_{\mathrm{x}}^{\mathrm{HF}} \\
& -\frac{\alpha}{2} \sum_{\substack{i j k l \\
\mathfrak{m}, \mathfrak{n} \in \mathcal{P}(I J)}} D_{j k} D_{i l}\left(R_{I, \mu}-R_{J, \mu}\right) \\
& \times\left[C_{i j}^{\mathrm{m}} \frac{\partial V_{\mathrm{mm}}}{\partial R_{I, \lambda}} C_{k l}^{\mathfrak{n}}+2 \frac{\partial C_{i j}^{\mathrm{m}}}{\partial R_{I, \lambda}} V_{\mathrm{mm}} C_{k l}^{\mathfrak{n}}\right] \\
& \sigma_{\lambda \mu}^{\mathrm{vdW}}=-\frac{1}{2 V} \sum_{I, J} \frac{C_{6 I J}}{R_{I J}^{6}} R_{I J, \mu}\left[\frac{\partial f_{\mathrm{damp}}\left(R_{I J}\right)}{\partial R_{I J, \lambda}}-6 \frac{R_{I J, \lambda}}{R_{I J}^{2}}\right]
\end{aligned}
$$


The strain derivative of the total electrostatic potential has been calculated before

$$
\begin{aligned}
\frac{\partial v_{\mathrm{es}}(\boldsymbol{r})}{\partial \varepsilon_{\lambda \mu}}=\sum_{I}[ & \frac{\partial v_{\mathrm{free}}^{I}\left(\boldsymbol{r}-\boldsymbol{R}_{I}\right)}{\partial \varepsilon_{\lambda \mu}}+\frac{\partial v_{\mathrm{NU}}^{I}\left(\boldsymbol{r}-\boldsymbol{R}_{I}\right)}{\partial \varepsilon_{\lambda \mu}} \\
& \left.+\frac{\partial v_{\mathrm{SR}}^{I}\left(\boldsymbol{r}-\boldsymbol{R}_{I}\right)}{\partial \varepsilon_{\lambda \mu}}+\frac{\partial v_{\mathrm{LR}}^{I}\left(\boldsymbol{r}-\boldsymbol{R}_{I}\right)}{\partial \varepsilon_{\lambda \mu}}\right]-\frac{\partial v_{\mathrm{avg}}}{\partial \varepsilon_{\lambda \mu}}
\end{aligned}
$$

and the individual derivatives can be looked up in Eq. 63, (64),

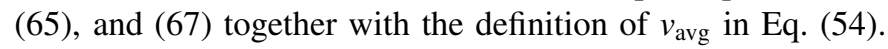
The strain derivatives of the orbitals $\psi_{i}$ and their gradients are given in Eq. (35) and (36), respectively.

For an actual computation, it is advisable to compute terms with the same bra and ket together to reduce the numerical effort. Furthermore, this practice avoids taking differences of large numbers and therefore leads to a reduction of numerical errors. In detail, this grouping means that the terms of the form $\left\langle\varphi_{j}|\ldots| \varphi_{k}\right\rangle$ of Eq. (96)/(97), (99), and (101)/(102)/(103) are computed together as one integral. The same is done for terms of the form $\left\langle\frac{\partial \varphi_{j}}{\partial \varepsilon_{\lambda \mu}}|\ldots| \varphi_{k}\right\rangle$ of Eq. 95, 96/ 97, ,99), and 101/ $/ 102 / 103$.

\section{Numerical implementation}

\subsection{Finite differences stress tensor}

In order to check the accuracy of the implemented analytical stress tensor, we calculate the stress tensor also numerically via finite differences of the total energies of distorted structures. Without accounting for space group symmetry, this requires the total energy calculation of twelve slightly distorted structures, i.e., two strain transformations $\varepsilon_{\lambda \mu}=\Delta h$ and $\varepsilon_{\lambda \mu}=-\Delta h$ for each of the six independent components of the stress tensor. The numerical derivative is then given by

$$
\sigma_{\lambda \mu}=\frac{1}{V} \frac{E_{\mathrm{tot}}\left(\varepsilon_{\lambda \mu}=\Delta h\right)-E_{\mathrm{tot}}\left(\varepsilon_{\lambda \mu}=-\Delta h\right)}{2 \Delta h}+O\left(\Delta h^{2}\right)
$$

On the one hand, the displacement $\Delta h$ must be small enough to ensure that the use of a two-point numerical derivative is justified. On the other hand, $\Delta h$ must not be too small at the same time. Otherwise, the differences become too small which leads to large numerical rounding errors. The convergence analysis with respect to the displacement $\Delta h$ shows that the value of $\Delta h=10^{-4}$ chosen for all calculations presented in this paper is reasonable. With this choice, we are in agreement with the literature [45], which proposes $\Delta h<10^{-2}$.

\subsection{Correction for sparse integration grids}

The calculation of the stress tensor involves integrals of quantities $f_{I}$ and $g_{I}$ and their derivatives that are centered on the same atom $I$, i.e., $\int d \boldsymbol{r} \frac{\partial f_{I}(\boldsymbol{r})}{\partial r_{j}} g_{I}(\boldsymbol{r})$. The integrands of these on-site terms are typically multiple orders of magnitude larger than those of off-site terms, especially for heavy elements. Accordingly, even minute relative numerical inaccuracies, e.g., due to relatively sparse integration grids used at light settings

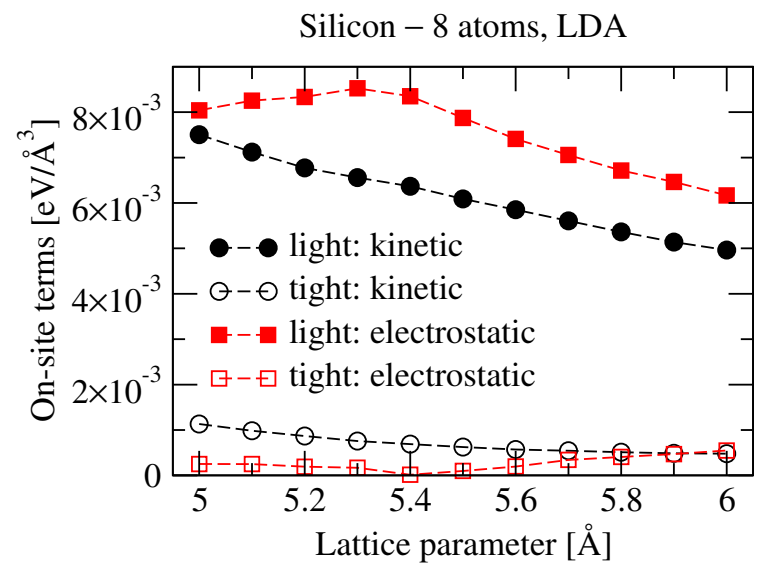

Figure 3: Absolute value of kinetic (circles) and electrostatic (squares) on-site terms at different lattice parameters. These terms are analytically zero but might be not in an actual numerical calculation and therefore, we omit them, see text. For light integration grids (filled symbols), the on-site terms are clearly nonzero whereas they become significantly smaller for tight grids (open symbols). A denser integration grid (going from light to tight) captures more accurately the overlap of the wave functions centered at the same atom. For more details about the used integration grid see Appendix D.1 The calculations were performed for a diamond unit cell of silicon (8 atoms) with the LDA functional.

(see Appendix D.1) or in the derivatives of the spherical harmonics, can result in notable absolute numerical inaccuracies for the stress tensor - even for a single on-site term. In the next two sections, we will point out the problematic terms and how we circumvent these issues.

\subsubsection{Kinetic on-site correction}

The term $\left\langle\psi_{i}\left|\hat{t}_{\mathrm{S}}\right| \psi_{i}\right\rangle$ of the non-relativistic kinetic energy (Eq. (38) ) can be numerically inaccurate for sparse integration grids. This inaccuracy stems from the on-site terms, i.e., the inner products of basis functions $j$ and $j^{\prime}$ located on the same atom $J$, namely

$$
T_{\mathrm{s}}^{\mathrm{on}-\mathrm{site}}=\sum_{i} \sum_{j, j^{\prime}} f_{i} c_{i j} c_{i j^{\prime}}\left\langle\varphi_{j}\left|\hat{t}_{\mathrm{s}}\right| \varphi_{j^{\prime}}\right\rangle
$$

Analytically, the strain derivative of this term should be zero since only quantities centered on one atom appear. Numerically, however, this is not always the case: Since both basis functions are located on the same atom, they have a huge overlap, and small numerical inaccuracies in such a huge term can effectively prevent these terms from vanishing as explained above. See Fig. 3 for a comparison between different integrations grids for the on-site term. To correct for this erroneous behavior, we omit the on-site term (Eq. (107) ) from Eq. (42) so that we get

$$
\frac{\partial T_{\mathrm{s}}}{\partial \varepsilon_{\lambda \mu}}=\delta_{\lambda \mu} T_{\mathrm{s}}+\sum_{\substack{i j k \\ J \neq K}} f_{i} c_{i j} c_{i k}\left[2\left\langle\frac{\partial \varphi_{j}}{\partial \varepsilon_{\lambda \mu}}\left|\hat{t}_{\mathrm{s}}\right| \varphi_{k}\right\rangle+\left\langle\varphi_{j}\left|\frac{\partial}{\partial r_{\lambda}} \frac{\partial}{\partial r_{\mu}}\right| \varphi_{k}\right\rangle\right] .
$$

Here, $J$ and $K$ specifies the atoms the basis function $j$ and $k$ are associated with, respectively, and it is enforced that atom $J$ is different from atom $K$. 


\subsubsection{Electrostatic on-site correction}

A similar problem occurs for the electrostatics. In Eq. (51), special care has to be taken for the strain derivative of the integral involving the term $n_{\mathrm{MP}}(\boldsymbol{r}) v_{\mathrm{es}}(\boldsymbol{r})$. One part of the total electrostatic potential includes the sum of the potentials of the free atoms $\sum_{I} v_{\text {free }}^{I}\left(\boldsymbol{r}-\boldsymbol{R}_{I}\right)(\mathrm{Eq}$. 55) $)$ and the multipole density is build up from the sum of the electron density of the free atoms $\sum_{I} n_{\text {free }}^{I}\left(\boldsymbol{r}-\boldsymbol{R}_{I}\right)$ and a residual part (Eq. 53). Hence, the total electrostatic energy in Eq. (51) involves a term that corresponds to the sum of the Hartree energy of the isolated, free atoms

$$
E_{\mathrm{es}, \text { free }}=-\frac{1}{2} \sum_{I} \int_{V} d \boldsymbol{r} n_{\text {free }}^{I}\left(\boldsymbol{r}-\boldsymbol{R}_{I}\right) v_{\text {free }}^{I}\left(\boldsymbol{r}-\boldsymbol{R}_{I}\right) .
$$

Analytically, the strain derivative of this term is zero since it includes only contributions of free atoms. For the exact same reasons detailed above, however, numerical inaccuracies can prevent this term from vanishing, especially for sparse integration grids. See Fig. 3 for a comparison between different integrations grids for this on-site term. To correct for this erroneous behavior, we explicitly leave out the strain derivative of the onsite term (Eq. (109), which is

$$
\begin{aligned}
\frac{\partial E_{\mathrm{es}, \mathrm{free}}}{\partial \varepsilon_{\lambda \mu}}= & \delta_{\lambda \mu} E_{\mathrm{es}, \mathrm{free}} \\
& -\frac{1}{2} \sum_{I} \int_{V} d \boldsymbol{r}\left(R_{I, \mu}-r_{\mu}\right) \\
& \times\left[n_{\mathrm{free}}^{I}\left(\boldsymbol{r}-\boldsymbol{R}_{I}\right) \frac{\partial v_{\mathrm{free}}^{I}\left(\boldsymbol{r}-\boldsymbol{R}_{I}\right)}{\partial R_{I, \lambda}}+\frac{\partial n_{\mathrm{free}}^{I}\left(\boldsymbol{r}-\boldsymbol{R}_{I}\right)}{\partial R_{I, \lambda}} v_{\text {free }}^{I}\left(\boldsymbol{r}-\boldsymbol{R}_{I}\right)\right]
\end{aligned}
$$

during the calculation of electrostatic contributions. Together with the correction for the kinetic energy in the previous section, this allows us to reduce the numerical inaccuracies by roughly one order of magnitude.

\section{Validation}

In this section, we perform an extensive comparison between the stress from finite differences (Sec.4.1), in short numerical stress, and the stress obtained from analytical gradients, in short analytical stress, for a large variety of crystal systems (Sec.5.1. Thereby, we mainly compare the diagonal elements but we consider the off-diagonal elements, too. In the following sections, we do further numerical tests by investigating the results for different exchange-correlation functionals (Sec. 5.2) and the consistency of the analytical stress with respect to the basis set and unit cell size (Sec. 5.3). Additionally, the performance of the implementation is examined (Sec. 5.5).

Unless noted otherwise, all calculations of the analytical stress tensor are performed with a partition table for the integrations that is build up from atom-centered partition functions following the recipe proposed by Delley [21]. The effect of this type of integration grid and another one, the so-called Stratmann partitioning [46], on the stress tensor is discussed below (Sec. 5.4).
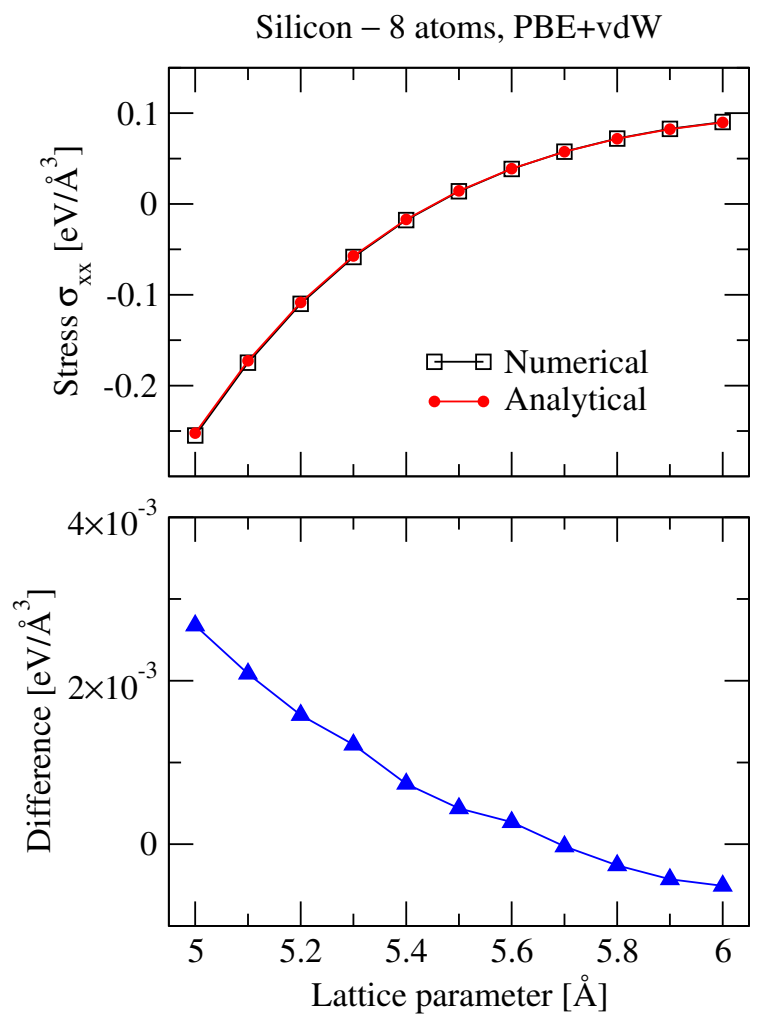

Figure 4: Comparison between numerical (black squares) and analytical (red circles) stress for a silicon crystal at different lattice parameters. The top figure shows the resulting stress for both types and the bottom one the difference between the two. The non-relativistic calculations were performed for a diamond unit cell of silicon (8 atoms) with tight settings, the PBE functional, and vdW corrections.

\subsection{Different crystal systems}

We test our analytical implementation with various different crystal systems and computational settings. At different lattice parameters around the equilibrium geometry, the numerical and analytical stress are computed by single point calculations. A plot of such calculations can be seen in Fig. 4 for silicon. If the crystal system has more than one lattice parameter, we vary each one separately while keeping the others fixed. The differences between the numerical and analytical stress are then evaluated by calculating the mean of these differences as well as the maximum of the absolute differences and the standard deviation.

In Tab. 1, the results for a broad range of crystal systems and computational settings are listed. We find that our analytical implementation is very accurate compared to the numerical stress for all tested crystal systems and exchange-correlation functionals. The mean difference, maximum absolute difference, and standard deviation of the difference between these two quantities is always in the range of $\mathrm{meV} / \AA^{3}$ or below, i.e., in the order of magnitude of the numerical error. This is substantiated by the fact that no constant offset, systematic variation, or regular pattern could be found in the respective deviations.

So far, we looked only at the diagonal elements of the stress tensor. We next address the off-diagonal elements at a exemplary system. In general, it suffices to calculate the upper 
Table 1: Mean difference, maximum absolute difference, and standard deviation of the difference between numerical and analytical stress for different crystal systems. All values are given in units of $m e V / \AA^{3}$. For the listed systems, the crystal and the total number of atoms in the unit cell is given. Under computational settings, the used exchange-correlation functional is noted as well as if a relativistic treatment was chosen (no entry means non-relativistic). Additionally, a collinear spin treatment was used for iron. It is also listed which lattice parameter is varied and which corresponding stress component is evaluated.

\begin{tabular}{|c|c|c|c|c|c|c|c|}
\hline Crystal & \#Atoms & Computational settings & Latt. par. & Stress Comp. & Mean diff. & Max. diff. & Std. dev. \\
\hline Aluminum (fcc) & 4 & PBE & $a$ & $x x$ & 0.6 & 3.1 & 1.3 \\
\hline Ammonia (cubic) & 16 & HSE06+vdW & $a$ & $x x$ & -0.3 & 0.4 & 0.1 \\
\hline \multirow[t]{3}{*}{ Benzene (orthorhombic) } & 48 & HSE06+vdW & $a$ & $x x$ & -0.4 & 0.9 & 0.5 \\
\hline & & & $b$ & $y y$ & -1.4 & 1.8 & 0.4 \\
\hline & & & $c$ & $z z$ & -0.1 & 0.6 & 0.5 \\
\hline Carbon dioxide (cubic) & 12 & HSE06+vdW & $a$ & $x x$ & -0.5 & 0.6 & 0.1 \\
\hline Gold (fcc) & 4 & PBE, rel. & $a$ & $x x$ & 0.6 & 2.2 & 0.7 \\
\hline \multirow[t]{2}{*}{ Graphite (hexagonal) } & 4 & $P B E+v d W$ & $a$ & $x x$ & -4.1 & 6.7 & 1.5 \\
\hline & & & $c$ & $z z$ & -0.9 & 1.3 & 0.2 \\
\hline Hexamine (bcc) & 22 & HSE06+vdW & $a$ & $x x$ & -0.2 & 0.2 & 0.0 \\
\hline \multirow[t]{2}{*}{ Iron (bcc) } & 1 & PBE, rel., coll. spin & $a$ & $x x$ & 0.0 & 0.3 & 0.1 \\
\hline & & PBE0, rel., coll. spin & $a$ & $x x$ & -0.5 & 6.5 & 3.0 \\
\hline \multirow[t]{3}{*}{ Naphthalene (monoclinic) } & 36 & HSE06+vdW & $a$ & $x x$ & -0.1 & 0.4 & 0.3 \\
\hline & & & $b$ & $y y$ & -1.1 & 1.8 & 0.7 \\
\hline & & & $c$ & $z z$ & -1.9 & 2.3 & 0.3 \\
\hline Sodium (bcc) & 2 & PBE & $a$ & $x x$ & -0.1 & 0.3 & 0.1 \\
\hline Sodium chloride (rock salt) & 2 & PBE & $a$ & $x x$ & 0.3 & 0.6 & 0.2 \\
\hline \multirow[t]{4}{*}{ Silicon (diamond) } & 8 & LDA & $a$ & $x x$ & 0.8 & 1.8 & 0.7 \\
\hline & & $\mathrm{PBE}+\mathrm{vdW}$ & $a$ & $x x$ & 0.7 & 2.7 & 1.1 \\
\hline & & PBE0 & $a$ & $x x$ & 1.0 & 2.7 & 0.9 \\
\hline & & HSE06 & $a$ & $x x$ & 1.0 & 2.7 & 0.9 \\
\hline \multirow[t]{2}{*}{ Silicon carbide (wurtzite) } & 4 & PBE & $a$ & $x x$ & 1.8 & 2.5 & 0.8 \\
\hline & & & $c$ & $z z$ & 0.2 & 0.8 & 0.5 \\
\hline \multirow[t]{2}{*}{ Urea (tetragonal) } & 16 & HSE06+vdW & $a$ & $x x$ & -0.2 & 0.4 & 0.1 \\
\hline & & & $c$ & $z z$ & -1.4 & 1.7 & 0.3 \\
\hline
\end{tabular}

Table 2: Difference between numerical and analytical stress in units of meV/ $\AA^{3}$ for different exchange-correlation functionals. The calculations were performed for a diamond unit cell of silicon (8 atoms) at a lattice parameter of $5.2 \AA$.

\begin{tabular}{|c|c|c|c|c|c|c|c|c|c|c|c|c|c|c|}
\hline & AM05 & B3LYP & BLYP & $\mathrm{HF}$ & HSE06 & PBE & PBE0 & PBEint & PBEsol & PBEsol0 & PW-LDA & PZ-LDA & revPBE & RPBE \\
\hline iff. & 1.4 & 1.9 & 1.8 & 1.8 & 1.8 & 1.8 & 1.8 & 1.4 & 1.8 & 1.8 & 1.5 & 1.4 & 1.7 & 1.7 \\
\hline
\end{tabular}

(lower) triangle of the stress tensor since it is symmetric by definition. However, to test the numerical accuracy of our implementation, we calculate the full stress tensor in this section and compare the off-diagonal elements. Fig. 5 shows that the off-diagonal elements of the analytical stress are very accurate, too. Additionally, the figure demonstrates that the difference between upper and lower triangle is vanishingly small.

\subsection{Different functionals}

As a further test, calculations with all LDA, GGA and hybrid exchange-correlation functionals available within FHI-aims were performed. The resulting difference between the numerical and analytical stress for a silicon crystal is listed in Tab. 2 The results show that our implementation is consistent across all tested functionals.

Additionally, for hybrid exchange correlation functionals, the fraction $\alpha$ of exact-exchange from Eq. (76) can be varied to check the accuracy of the analytical stress. For a silicon crystal, the difference to the numerical stress stays almost constant, see Fig. 6, and scales only weakly with $\alpha$ showing that we have a consistent implementation.

\subsection{Basis set and unit cell size}

In this section, we demonstrate the consistency of the analytical stress with respect to the basis set and unit cell size. The difference between numerical and analytical stress stays almost constant when the basis set size is increased, see Fig. 7 . Fig. 8 shows that the analytical stress stays constant within numerical limitations when the size of the unit cell is increased.

\subsection{Partition functions for integration}

In FHI-aims, the numerical integrations are performed by partitioning the integrand with the help of atom-centered partition functions [21, 22, 46], similarly to the partitioning of the 


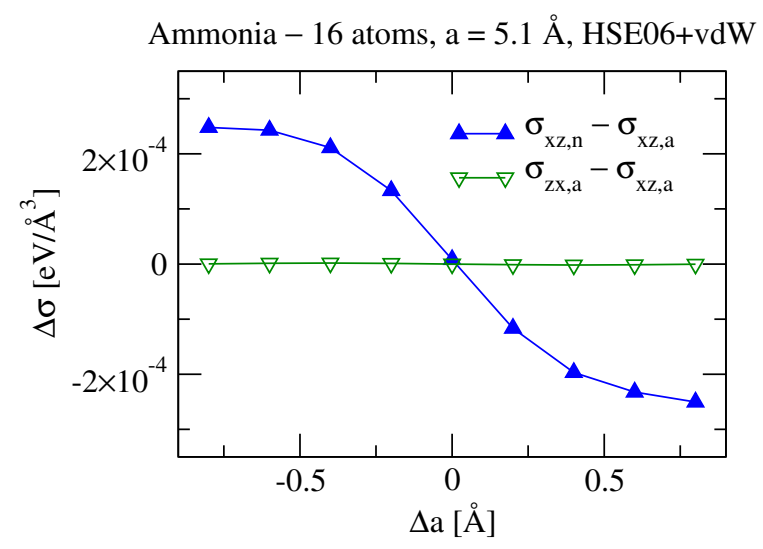

Figure 5: Off-diagonal stress for a distorted cubic unit cell with lattice vectors $(a, 0, \Delta a),(0, a, 0)$, and $(\Delta a, 0, a)$. Blue, filled triangles: Difference between the off-diagonal elements of numerical $\left(\sigma_{x z, n}\right)$ and analytical $\left(\sigma_{x z, a}\right)$ stress. Green, open triangles: Difference between the off-diagonal elements of upper $\left(\sigma_{x z, a}\right)$ and lower $\left(\sigma_{z x, a}\right)$ triangle of the analytical stress. The calculations were performed for a unit cell of ammonia (16 atoms) with the HSE06 functional and vdW corrections at a lattice parameter of $5.1 \AA$.

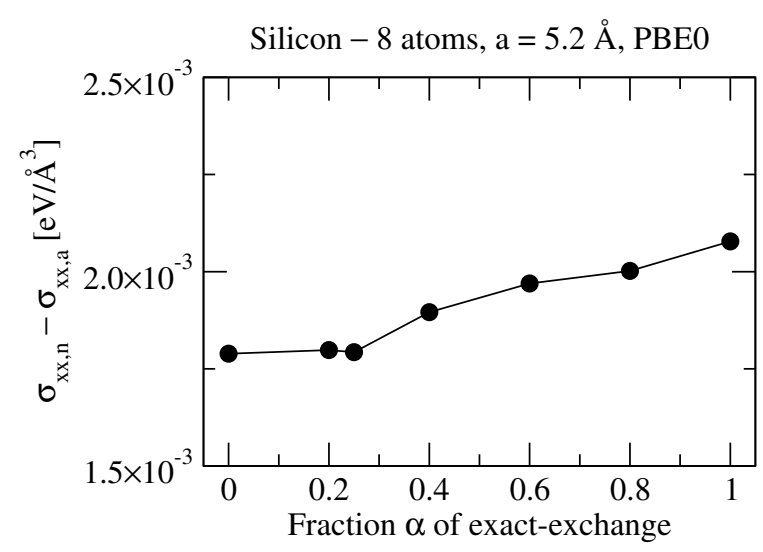

Figure 6: Difference between numerical $\left(\sigma_{x x, n}\right)$ and analytical $\left(\sigma_{x x, a}\right)$ stress for different fractions $\alpha$ of exact-exchange. The calculations were performed for a diamond unit cell of silicon ( 8 atoms) with the PBE0 functional at a lattice parameter of $5.2 \AA$.

electronic density as described in Sec. 3.4 With this, an integral can be rewritten in the following way

$$
\int d \boldsymbol{r} f(\boldsymbol{r})=\sum_{I} \int d \boldsymbol{r} p_{I}(\boldsymbol{r}) f(\boldsymbol{r}),
$$

where the atom-centered partition functions $p_{I}(\boldsymbol{r})$ are defined as

$$
p_{I}(\boldsymbol{r})=\frac{g_{I}(\boldsymbol{r})}{\sum_{J} g_{J}(\boldsymbol{r})},
$$

and $g_{I}(\boldsymbol{r})$ is an in principle arbitrary function that is strongly peaked at atom $I$. In general, these functions change under strain, and therefore, contribute to the stress tensor. However, in the limit of an infinitively dense integration grid, this additional contribution vanishes since such a grid stays constant under a strain transformation.

The $n / r^{2}$-type partition function by Delley [21] uses $g_{I}(\boldsymbol{r})=$ $n_{I}^{\text {free }}(\boldsymbol{r}) /|\boldsymbol{r}|^{2}$ and $n_{I}^{\text {free }}(\boldsymbol{r})$ is the electron density of the free

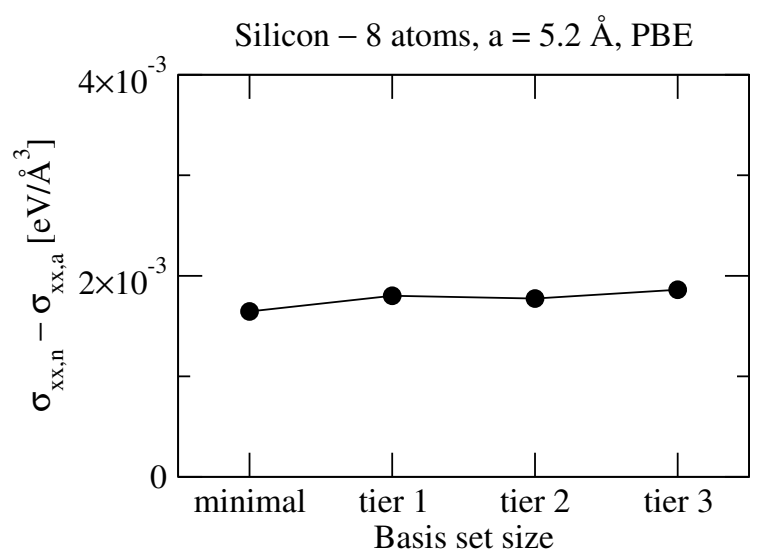

Figure 7: Difference between numerical $\left(\sigma_{x x, n}\right)$ and analytical $\left(\sigma_{x x, a}\right)$ stress for increasing basis set size. The higher the tier in FHI-aims, the more basis functions are included. For more details about the used basis set see Appendix D.2 The calculations were performed for a diamond unit cell of silicon (8 atoms) with the PBE functional at a lattice parameter of $5.2 \AA$.

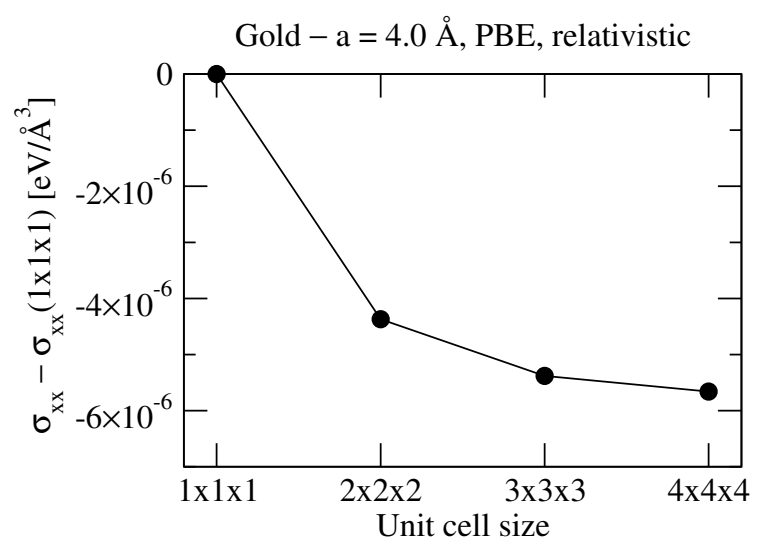

Figure 8: Change of the analytical stress for increasing unit cell sizes relative to a $1 \times 1 \times 1$ unit cell ( 1 atom). The calculations were performed for fcc unit cells of gold with the PBE functional and relativistic treatment at a lattice parameter of $4.0 \AA$.

atom 13 For such a partition function the contribution to the stress tensor will be small because the partition functions move equally with the atoms under a strain transformation and certain integrands are built up from atom-centered quantities. Fig. 9 shows that the contribution of the integration grid for a $n / r^{2}-$ type partition function is considerably below meV/ $\AA^{3}$ (thus, below the numerical accuracy) and the analytical stress converges rapidly towards a certain value by increasing the grid density.

We expect to get bigger contributions to the stress tensor compared to the $n / r^{2}$-type partition function, if the partition function depends on the position of the atoms with respect to each other. For instance, this is the case for a Stratmann partitioning scheme [46], a modified version of which has been implemented in FHI-aims (see Appendix C). However, Fig. 9 shows that the contribution of the "modified Stratmann" partition function is on the same order as for the $n / r^{2}$-type parti-

\footnotetext{
${ }^{3}$ In the case of FHI-aims, the confined free atom electron density given by the confining potential $v_{\text {cut }}$ of Eq. (9) in Ref. [16] is used for the partition function and electrostatics.
} 
Silicon -8 atoms, a $=5.2 \AA$, LDA

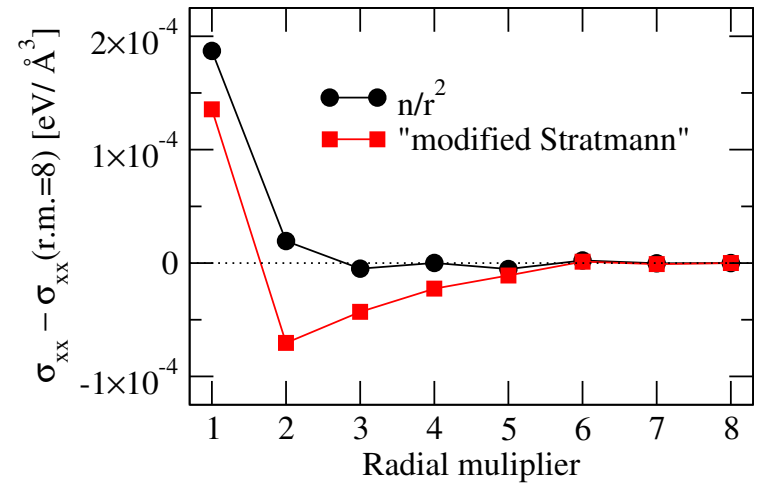

Figure 9: Change of the analytical stress for increasing integration grid densities relative to the densest used grid. The behavior for two different integration partition functions is shown, see text. Increasing the radial multiplier increases the number of spherical integration shells around every atom, and therefore, leading to a denser integration grid. For more details about the used integration grid see Appendix D.1 The calculations were performed for a diamond unit cell of silicon (8 atoms) with the LDA functional at a lattice parameter of $5.2 \AA$.

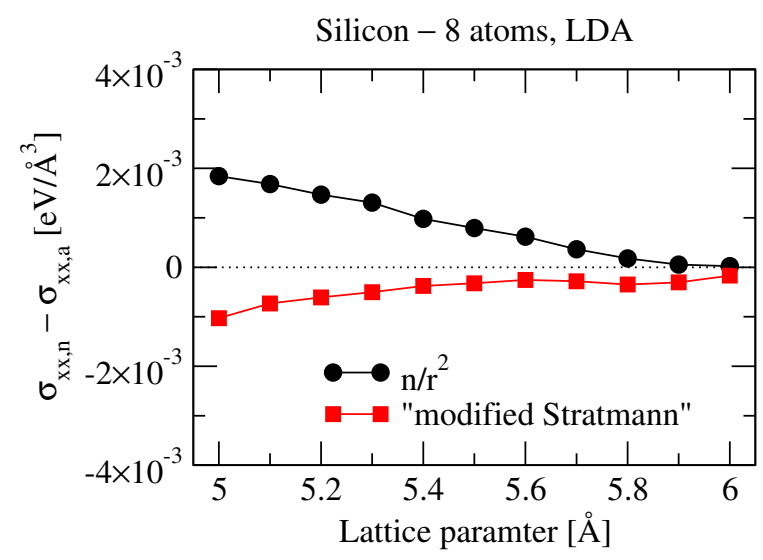

Figure 10: Difference between numerical $\left(\sigma_{x x, n}\right)$ and analytical $\left(\sigma_{x x, a}\right)$ stress for two different integration partition functions, see text. The calculations were performed for a diamond unit cell of silicon ( 8 atoms) with the LDA functional.

tion function. The only difference is that the analytical stress converges more slowly with increasing integration grid density, which is insignificant since the differences are below the target numerical accuracy required for practical calculations.

In summary, the contribution of the integration grid to the stress tensor are very small (considerably below $\mathrm{meV} / \AA^{3}$ ), and we therefore neglect this contribution. Also, using a Stratmann partition function does not lead to larger deviations in the difference between numerical and analytical stress compared to a $n / r^{2}$-type partition function if we do calculations for different lattice parameters (Fig. 10).

\subsection{Timings}

In this section, the efficiency of our analytical stress implementation is estimated. The exact numbers are specific to our implementation but can be seen as a guidance. In order to test the performance of the computation of the analytical stress, we compare the computing time for total energy, forces, analytical
Silicon -8 atoms, $\mathrm{a}=5.2 \AA$

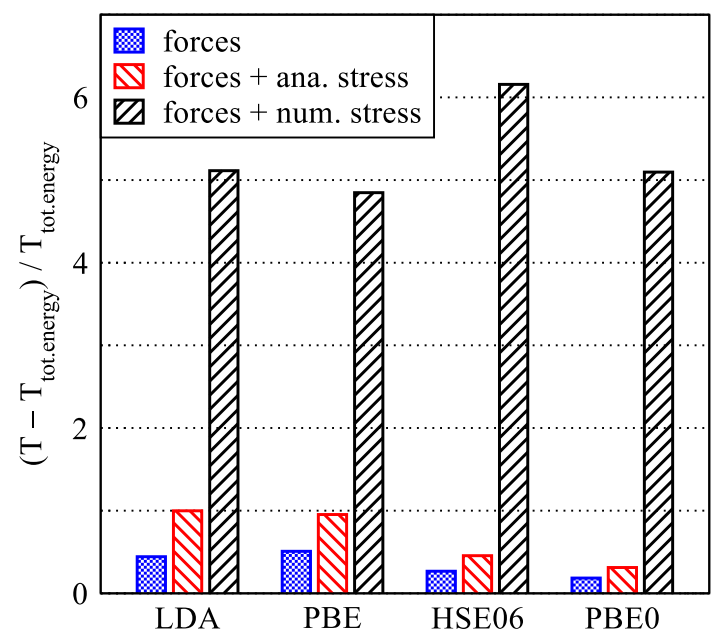

Figure 11: Comparison between the computing time for forces, forces + analytical stress, and forces + numerical stress for different exchange-correlation functionals. We take the difference between the time $T_{\text {tot.energy }}$ for a total energy calculation and time $T$ for a calculation of one of the quantities listed before. Then, the value relative to $T_{\text {tot.energy }}$ is plotted. The calculations were performed for a diamond unit cell of silicon ( 8 atoms) at a lattice parameter of $5.2 \AA$.

stress, and numerical stress. For this purpose, we measure how much additional time the computation takes relative to the time for a total energy computation. For LDA and PBE, the computation of the analytical stress including forces takes roughly double the time of a total energy computation, see Fig. 11. The time in addition to a total energy computation is only doubled compared to the computation of forces. This is a very good result if one considers that three derivatives have to be calculated for the forces, however, for the analytical stress including forces, nine derivatives (three for forces and six for stress) are needed. The additional cost for the computation of the analytical stress including forces is significantly lower for hybrid functionals since several terms of the exact-exchange energy computation can be reused (compare Eq. 82 and (89) and the evaluation of the exact-exchange part dominates the overall computing time.

The additional time for the computation of the numerical stress including forces is roughly five to six times the time for a total energy calculation for all tested exchange correlation functionals, see Fig. 11. While this might be still computationally feasible for LDA and GGA functionals, it is definitely a heavy burden for unit cell optimizations with hybrid functionals.

Fig.12.a) exemplifies the typical computational performance and scaling of the implemented strain derivatives for the electrostatic potential as function of the system size. In agreement with previous studies [16, 18], we find almost linear scaling $O\left(N^{1.3}\right)$ with respect to the number of atoms $N$ for the total electrostatic potential, in spite of the fact that the terms that are computed in reciprocal space scale almost quadratically $O\left(N^{1.9}\right)$. Formally, we expect such a scaling since in addition to the operations on the real space grid which scale as $O(N)$ [16] a sum over the $\boldsymbol{G}$-vectors occurs in Eq. (58). In turn, this sum scales linearly with the volume of the unit cell/the 


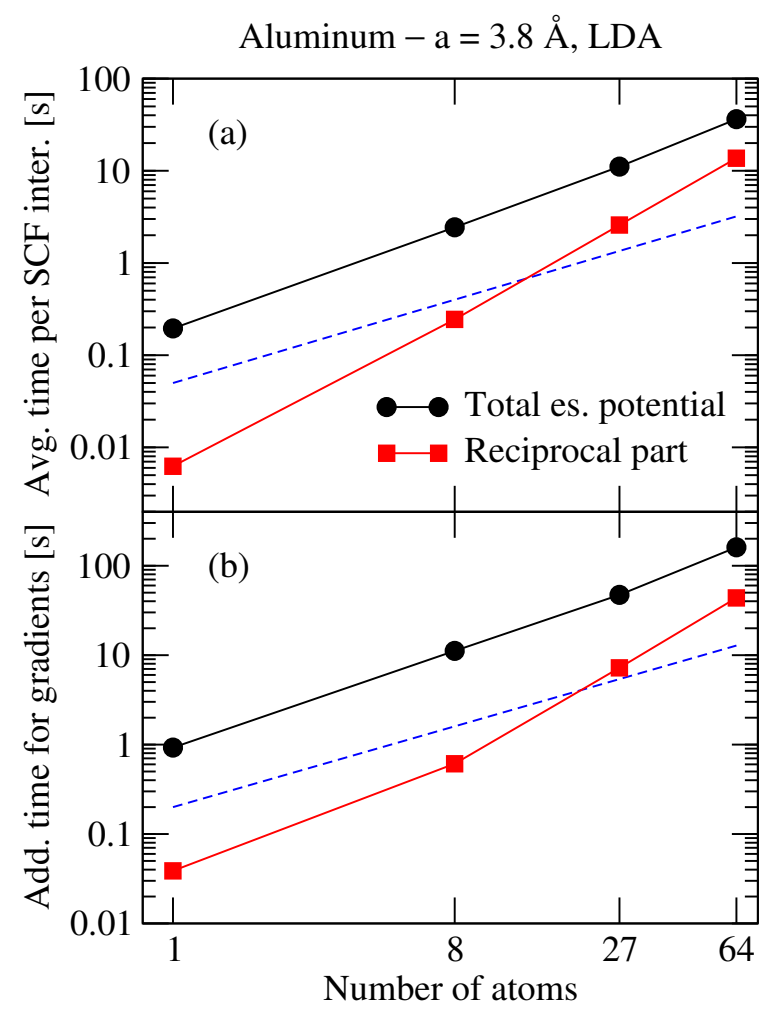

Figure 12: Average wall clock time (per SCF iteration on 36 CPU cores, fcc aluminum, LDA, lattice constant $3.8 \AA$ ) for the computation of the total electrostatic potential (black) and of the reciprocal part (red) as function of the system size. To guide the eye, the dashed blue lines exemplify an ideal linear scaling. The upper plot (a) shows the wall time needed to evaluate all terms required to compute the total electrostatic energy, whereas the lower plot (b) shows the additional wall time required to compute the respective gradients (forces and stress).

number of atoms $N$. Due to the minute prefactor, however, the reciprocal term does not dominate the scaling for system sizes studied here (below $\sim 200$ atoms, not shown in Fig. 12, so that we find approximately $O(N)$ scaling as originally discussed by Delley [18]. Fig. 12 b) shows that the calculation of analytical gradients (forces and stress) only affects the prefactors, but not the overall scaling behavior with respect to system size $\left(O\left(N^{1.3}\right)\right.$ for all electrostatic terms and $O\left(N^{2.1}\right)$ for the terms computed in reciprocal space). As a consequence, even for large systems $(N=125$, not shown in Fig. 12) the computation of the electrostatics together with its gradients only takes up a fraction $(<16 \%)$ of the total computational time of a full self-consistent field (SCF) cycle.

\section{Unit cell optimization and pressure}

For the sake of completeness, we sketch briefly how the stress tensor can be used to optimize the unit cell of a crystal structure. For this, the forces acting on the lattice vectors $\boldsymbol{a}_{n}$ are needed which we define as the negative derivative of the total energy $E_{\text {tot }}$ with respect to the lattice vectors

$$
\boldsymbol{F}_{n}^{\text {lat }}=-\frac{\partial E_{\mathrm{tot}}}{\partial \boldsymbol{a}_{n}}
$$

Since the stress tensor depends only on the atom positions and the lattice vectors, Eq. (13) reads as

$$
V \sigma_{\lambda \mu}=\sum_{I} \frac{\partial E_{\mathrm{tot}}}{\partial R_{I, \lambda}} R_{I, \mu}+\sum_{n} \frac{\partial E_{\mathrm{tot}}}{\partial a_{\lambda n}} a_{\mu n},
$$

where the strain derivatives have already been evaluated according to Sec. 2.2. The derivatives of the total energy with respect to the atom positions are the atomic forces, $F_{I, \lambda}=-\frac{\partial E_{\mathrm{tot}}}{\partial R_{I, \lambda}}$. Here, $F_{I, \lambda}$ is component $\lambda$ of the force acting on atom $I$. Solving Eq. (114) for the lattice vector derivative yields the forces acting on the lattice vectors, namely,

$$
F_{\lambda n}^{\text {lat }}=-\sum_{\mu} V \sigma_{\lambda \mu}\left(A^{-1}\right)_{n \mu}-\sum_{\mu, I} F_{I, \lambda} R_{I, \mu}\left(A^{-1}\right)_{n \mu}
$$

with $A$ the matrix of the lattice vectors, see Sec. 2.2.3. $F_{\lambda n}^{\text {lat }}$ is component $\lambda$ of the force acting on lattice vector $\boldsymbol{a}_{n}$.

Together with the atomic forces, the lattice vector forces can be used to optimize the structure of the unit cell and the position of the atoms of a crystal. In order to treat atoms and lattice vectors on equal footing, we define a generalized coordinate vector, $\boldsymbol{X}=\left\{a_{x 1}, a_{y 1}, \ldots, a_{z 3}, R_{1, x}, R_{1, y}, \ldots, R_{N, z}\right\}$, containing the lattice vectors and atom positions. In addition, we can write the corresponding generalized force vector as $\boldsymbol{F}=\left\{F_{x 1}^{\text {lat }}, F_{y 1}^{\text {lat }}, \ldots, F_{z 3}^{\text {lat }}, F_{1, x}, F_{1, y}, \ldots, F_{N, z}\right\}$, containing the lattice vector forces and atomic forces. Together with $E_{\text {tot }}, X$ and $\boldsymbol{F}$ can be used as an input for an optimization scheme to obtain the local minimum of the crystal structure.

For the optimization an initial guess for the Hessian is needed. In the case of FHI-aims, the Lindh model matrix [47] is used to initialize the Hessian between all atomic coordinates. Since we did not implement the Lindh method for periodic boundary conditions, we set all the Hessian rows and columns associated with the lattice vector degrees of freedom to be diagonal. The diagonal entries are chosen to have a conservative value of $25 \mathrm{eV} / \AA^{2}$.

With the analytical stress tensor and the finite difference infrastructure described above at hand, it is alternatively possible to determine the Hessian of the lattice vectors by finite differences (FD). This requires 18 (three lattice vectors, three coordinates, two distortions for central finite difference) separate analytical stress calculations, if the Hessian is determined by using finite differences of the forces acting on the lattice vectors. Quite surprisingly, using this FD Hessian of the lattice vectors as an input for the relaxation does not reduce the number of required optimization steps considerably, as Tab. 3 shows for two representative test cases. We could trace back this finding to the fact that the cross-terms, i.e., the entries of the Hessian that are mixed derivatives with respect to lattice and atomic degrees of freedom, are still zero, as substantiated in Tab. 3 with the following strategy: During the geometry optimization, the Hessian matrix is updated at each step according to the Broyden-Fletcher-Goldfarb-Shanno (BFGS) formula [48], as a consequence of which an approximate Hessian at the local minimum is known at the end of the optimization. We now use parts of this BFGS Hessian obtained by a previous optimization 
Table 3: Required optimization steps for the relaxation of monoclinic naphthalene $(\mathrm{PBE}+\mathrm{vdW})$ for different choices of the initial Hessians (see text) for the lattice vectors, the atoms, and the cross-terms. For case (a), the initial geometry corresponds to an uniformly $25 \%$ increased equilibrium unit cell. For case (b), the monoclinic angle was increase by $16^{\circ}$ and one lattice vector was shortened by $10 \%$ with respect to equilibrium.

\begin{tabular}{ccc|cc}
\hline latt. vec. & atoms & cross-terms & (a) volume & (b) angle \\
\hline diag. & Lindh & $0^{*}$ & 76 & 117 \\
FD & Lindh & 0 & 69 & 111 \\
FD & BFGS & 0 & 64 & 69 \\
BFGS & BFGS & 0 & 77 & 78 \\
BFGS & BFGS & BFGS & 18 & 45 \\
\hline
\end{tabular}

*This is the standard Hessian of FHI-aims.

to investigate which terms (lattice, atoms, or cross-terms) influence the performance of the optimization mos ${ }^{4}$. Obviously, the optimization is sped up greatly if the full BFGS Hessian is presented to the relaxation algorithm from the beginning (last line in Tab. 3). A brute force numerical calculation of the full Hessian for both lattice and atomic degrees of freedom with finite differences requires $6(N+3)$ separate calculations of the gradients (forces and stress) with $N$ the number of atoms and is thus not particularly useful in practice. However, also feeding only parts of this BFGS Hessian to the relaxation algorithm without the cross terms has no considerable impact on the number of relaxation steps. In summary, these investigations show that (a) a more accurate estimate of the lattice vector Hessian is not generally required (but might be useful in corner cases) and (b) that more educated guesses (in the spirit of [47]) for the full Hessian that must include the cross-terms have the potential to speed up combined volume/geometry relaxations considerably.

The above described method to optimize crystal structures can be extended to the case where external pressure is acting on the crystal. When we apply external hydrostatic pressure $p_{\text {ext }}$ to the crystal, then, we have to minimize the enthalpy $H(S, p)$ instead of the total (inner) energy $E_{\text {tot }}(S, V)$ of the underlying system. The enthalpy is given by

$$
H(S, p)=E_{\text {tot }}(S, V)+p_{\text {ext }} V
$$

with $V$ the volume of the system. We see immediately that the atomic forces are not affected by the external pressure, however, we get an additional contribution to the stress tensor since the unit cell volume depends on the strain. Therefore, the total stress is given by

$$
\sigma_{\lambda \mu}^{\mathrm{tot}}=\sigma_{\lambda \mu}+\delta_{\lambda \mu} p_{\mathrm{ext}},
$$

where $\sigma_{\lambda \mu}$ is given in Sec. 3.6 and the contribution of the external pressure was evaluated according to Eq. (19).

Finding the local minimum under pressure works exactly as described above, only $\sigma_{\lambda \mu}$ in Eq. 115 has to replaced by $\sigma_{\lambda \mu}^{\text {tot }}$ from Eq. (117). Then, $H, \boldsymbol{X}$, and $\boldsymbol{F}$ can be used for the optimization scheme.

\footnotetext{
${ }^{4}$ Evidently, this strategy is not useful in practice since it requires knowledge of the equilibrium geometry that is actually sought after.
}

\section{Conclusions}

In this paper, we have derived the stress tensor in an all-electron, numeric atom-centered orbital based densityfunctional formalism. We have presented all relevant contributions to the stress including those from hybrid exchangecorrelation functionals and from a pairwise van der Waals correction scheme. Furthermore, we have given details about corrections for the numerical calculations in order to obtain more accurate results. We have validated that our implementation in the electronic structure theory code FHI-aims works and produces consistent results at different levels of theory and accuracy, e.g., for various exchange-correlation functionals, basis set sizes, system sizes, and integration grids. The resulting accuracy has been investigated for a wide range of crystal systems. Thereby, the difference between the stress tensor calculated via our analytical derivatives and the one calculated via finite differences is always in the range of $\mathrm{meV} / \AA^{3}$ or below which is comparable to the magnitude of the numerical error. This holds even true for the lowest computational settings (light) which employ a rather sparse integration grid. In comparison to the finite difference method, the calculation of the stress tensor with our implementation allows a speedup of at least factor three up to five for hybrid exchange-correlation functionals. Therefore, our method allows us to perform accurate and rapid optimization of crystal structures under pressure.

\section{Acknowledgments}

The authors acknowledge fruitful discussions with Jürgen Wieferink. We thank the unknown referee for suggesting the monoclinic naphtalene testcase in Sec. 6. Christian Carbogno acknowledges funding from the DFG (CA 1088/1-1).

\section{Appendix A. Strain derivatives of electrostatics}

\section{Appendix A.1. Strain derivative of on-site Hartree term}

We want to show that the strain derivative of the Hartree potential $v_{\mathrm{H}}^{I}\left(\boldsymbol{r}-\boldsymbol{R}_{I}\right)$ vanishes for $\boldsymbol{r}=\boldsymbol{R}_{I}$. Starting with the definition from Eq. (49) and using Eq. (15) and Eq. (34), we get

$$
\begin{aligned}
\frac{\partial v_{\mathrm{H}}^{I}\left(\boldsymbol{r}-\boldsymbol{R}_{I}\right)}{\partial \varepsilon_{\lambda \mu}}= & \delta_{\lambda \mu} v_{\mathrm{H}}^{I}\left(\boldsymbol{r}-\boldsymbol{R}_{I}\right) \\
& +\int_{V} d \tilde{\boldsymbol{r}}\left(\tilde{r}_{\mu}-R_{I, \mu}\right) \frac{\partial n_{I}\left(\tilde{\boldsymbol{r}}-\boldsymbol{R}_{I}\right)}{\partial \tilde{r}_{\lambda}} \frac{1}{|\tilde{\boldsymbol{r}}-\boldsymbol{r}|} \\
& +\int_{V} d \tilde{\boldsymbol{r}}\left(\tilde{r}_{\mu}-r_{\mu}\right) n_{I}\left(\tilde{\boldsymbol{r}}-\boldsymbol{R}_{I}\right) \frac{\partial}{\partial \tilde{r}_{\lambda}} \frac{1}{|\tilde{\boldsymbol{r}}-\boldsymbol{r}|} .
\end{aligned}
$$

Next, we use integration by parts on the third term and the boundary term vanishes on the surface of the unit cell. Together with the product rule of derivatives, we obtain

$$
\begin{aligned}
\int_{V} d \tilde{\boldsymbol{r}}\left(\tilde{r}_{\mu}-\right. & \left.r_{\mu}\right) n_{I}\left(\tilde{\boldsymbol{r}}-\boldsymbol{R}_{I}\right) \frac{\partial}{\partial \tilde{r}_{\lambda}} \frac{1}{|\tilde{\boldsymbol{r}}-\boldsymbol{r}|} \\
= & -\delta_{\lambda \mu} v_{\mathrm{H}}^{I}\left(\boldsymbol{r}-\boldsymbol{R}_{I}\right) \\
& -\int_{V} d \tilde{\boldsymbol{r}}\left(\tilde{r}_{\mu}-r_{\mu}\right) \frac{\partial n_{I}\left(\tilde{\boldsymbol{r}}-\boldsymbol{R}_{I}\right)}{\partial \tilde{r}_{\lambda}} \frac{1}{|\tilde{\boldsymbol{r}}-\boldsymbol{r}|} .
\end{aligned}
$$


We see that the two delta terms cancel each other. In the case $\boldsymbol{r}=\boldsymbol{R}_{I}$, the two integrals cancel each other, too, and the strain derivative vanishes.

\section{Appendix A.2. Strain derivative of Ewald long range term}

Here, we show the individual derivatives of the Ewald long range term, see Eq. 66. The derivatives with respect to the position vectors are

$$
\begin{array}{r}
\frac{\partial v_{\mathrm{LR}}^{I}\left(\boldsymbol{r}-\boldsymbol{R}_{I}\right)}{\partial r_{\lambda}} r_{\mu}=\frac{4 \pi}{V} \sum_{G} i G_{\lambda} r_{\mu} \frac{\exp \left(-\frac{\kappa^{2} G^{2}}{4}\right)}{G^{2}} \\
\times S_{I}(\boldsymbol{G}) \exp (i \boldsymbol{G r}), \\
\frac{v_{\mathrm{LR}}^{I}\left(\boldsymbol{r}-\boldsymbol{R}_{I}\right)}{\partial R_{I, \lambda}} R_{I, \mu}=-\frac{4 \pi}{V} \sum_{G} i G_{\lambda} R_{I, \mu} \frac{\exp \left(-\frac{\kappa^{2} G^{2}}{4}\right)}{G^{2}} \\
\times S_{I}(\boldsymbol{G}) \exp (i \boldsymbol{G} \boldsymbol{r}),
\end{array}
$$

and the volume derivative is

$$
\frac{\partial v_{\mathrm{LR}}^{I}\left(\boldsymbol{r}-\boldsymbol{R}_{I}\right)}{\partial V}=-\frac{1}{V} v_{\mathrm{LR}}^{I}\left(\boldsymbol{r}-\boldsymbol{R}_{I}\right) .
$$

For the $G$-vector, we need the following derivatives

$$
\frac{\partial S_{I}(\boldsymbol{G})}{\partial G_{\mu}}=\Gamma_{I, \mu}(\boldsymbol{G})-i R_{I, \mu} S_{I}(\boldsymbol{G})
$$

and

$$
\frac{\partial}{\partial G_{\lambda}}\left(\frac{\exp \left(-\frac{\kappa^{2} G^{2}}{4}\right)}{G^{2}}\right)=-\frac{\exp \left(-\frac{\kappa^{2} G^{2}}{4}\right)}{G^{2}}\left(\frac{2}{G^{2}}+\frac{\kappa^{2}}{2}\right) G_{\lambda} .
$$

The definitions of $S_{I}(\boldsymbol{G})$ and $\Gamma_{I, \mu}(\boldsymbol{G})$ are given in Eq. 59) and Eq. (69), respectively. With this, the derivative of the Ewald long range term with respect to the $G$-vector is

$$
\begin{aligned}
\frac{\partial v_{\mathrm{LR}}^{I}\left(\boldsymbol{r}-\boldsymbol{R}_{I}\right)}{\partial G_{\mu}} G_{\lambda}= & \frac{4 \pi}{V} \sum_{k} \frac{\exp \left(-\frac{\kappa^{2} G^{2}}{4}\right)}{G^{2}} \exp (i \boldsymbol{G r}) G_{\lambda} \\
\times & {\left[G_{\mu} S_{I}(\boldsymbol{G})\left(\frac{2}{G^{2}}+\frac{\kappa^{2}}{2}\right)+\Gamma_{I, \mu}(\boldsymbol{G})\right.} \\
& \left.+i r_{\mu} S_{I}(\boldsymbol{G})-i R_{I, \mu} S_{I}(\boldsymbol{G})\right]
\end{aligned}
$$

Since for the strain derivative of $v_{\mathrm{LR}}(\boldsymbol{r})$ the negative of the $\boldsymbol{G}$ vector derivative is needed (Eq. (66) ), the terms of the position vectors derivative cancel completely with the third and fourth term of Eq. A.8.

\section{Appendix B. Strain derivatives of exact exchange}

\section{Appendix B.1. Strain derivative of Coulomb matrix}

We want to show that the derivative of the Coulomb matrix can be changed from $\frac{\partial}{\partial R_{I, \lambda}}$ to $-\frac{\partial}{\partial R_{J, \lambda}}$. We will use the notation $\mathrm{m}(I)$ to indicate that the auxiliary basis function $\mathrm{m}$ is centered on atom $I$. In addition, we will employ integration by parts (IP) and the boundary terms vanish for the Coulomb integral since the basis functions are zero at infinity:

$$
\begin{aligned}
\frac{\partial V_{\mathfrak{m}(I) \mathfrak{n}(J)}}{\partial R_{I, \lambda}} & =\iint d \boldsymbol{r} d \tilde{\boldsymbol{r}} \frac{\partial P_{\mathfrak{m}}\left(\boldsymbol{r}-\boldsymbol{R}_{I}\right)}{\partial R_{I, \lambda}} v(|\boldsymbol{r}-\tilde{\boldsymbol{r}}|) P_{\mathfrak{n}}\left(\tilde{\boldsymbol{r}}-\boldsymbol{R}_{J}\right) \\
& =-\iint d \boldsymbol{r} d \tilde{\boldsymbol{r}} \frac{\partial P_{\mathfrak{m}}\left(\boldsymbol{r}-\boldsymbol{R}_{I}\right)}{\partial r_{\lambda}} v(|\boldsymbol{r}-\tilde{\boldsymbol{r}}|) P_{\mathfrak{n}}\left(\tilde{\boldsymbol{r}}-\boldsymbol{R}_{J}\right) \\
& \stackrel{I P}{=} \iint d \boldsymbol{r} d \tilde{\boldsymbol{r}} P_{\mathfrak{m}}\left(\boldsymbol{r}-\boldsymbol{R}_{I}\right) \frac{\partial v(|\boldsymbol{r}-\tilde{\boldsymbol{r}}|)}{\partial r_{\lambda}} P_{\mathfrak{n}}\left(\tilde{\boldsymbol{r}}-\boldsymbol{R}_{J}\right) \\
& =-\iint d \boldsymbol{r} d \tilde{\boldsymbol{r}} P_{\mathfrak{m}}\left(\boldsymbol{r}-\boldsymbol{R}_{I}\right) \frac{\partial v(|\boldsymbol{r}-\tilde{\boldsymbol{r}}|)}{\partial \tilde{r}_{\lambda}} P_{\mathfrak{n}}\left(\tilde{\boldsymbol{r}}-\boldsymbol{R}_{J}\right) \\
& \stackrel{I P}{=} \iint d \boldsymbol{r} d \tilde{\boldsymbol{r}} P_{\mathfrak{m}}\left(\boldsymbol{r}-\boldsymbol{R}_{I}\right) v(|\boldsymbol{r}-\tilde{\boldsymbol{r}}|) \frac{\partial P_{\mathfrak{n}}\left(\tilde{\boldsymbol{r}}-\boldsymbol{R}_{J}\right)}{\partial \tilde{r}_{\lambda}} \\
& =-\iint d \boldsymbol{r} d \tilde{\boldsymbol{r}} P_{\mathfrak{m}}\left(\boldsymbol{r}-\boldsymbol{R}_{I}\right) v(|\boldsymbol{r}-\tilde{\boldsymbol{r}}|) \frac{\partial P_{\mathfrak{n}}\left(\tilde{\boldsymbol{r}}-\boldsymbol{R}_{J}\right)}{\partial R_{J, \lambda}} \\
& =-\frac{\partial V_{\mathfrak{m}(I) \mathfrak{n}(J)} .}{\partial R_{J, \lambda}} .
\end{aligned}
$$

Appendix B.2. Strain derivative of expansion coefficients

We want to show that the derivative of the expansion coefficients can be changed from $\frac{\partial}{\partial R_{I, \lambda}}$ to $-\frac{\partial}{\partial R_{J, \lambda}}$. We will use the notation $i(I)$ to indicate that the basis function $i$ is centered on atom $I$. In addition, we will employ integration by parts (IP) and the boundary terms vanish for the Coulomb integrals since the basis functions are zero at infinity.

First, we will separately show that the derivative for each of the two factors $(i j \mid \mathfrak{n})$ and $L_{\mathfrak{r m}}^{I J}$ of Eq. 85 can be changed and we begin with the Coulomb integral for the case that $n$ is centered on atom $I$ :

$$
\begin{aligned}
\frac{\partial(i(I)}{\partial(J) \mid \mathfrak{n}(I))} & \partial R_{I, \lambda} \\
= & \iint d \boldsymbol{r} d \tilde{\boldsymbol{r}} \frac{\partial \varphi_{i}\left(\boldsymbol{r}-\boldsymbol{R}_{I}\right)}{\partial R_{I, \lambda}} \varphi_{j}\left(\boldsymbol{r}-\boldsymbol{R}_{J}\right) v(|\boldsymbol{r}-\tilde{\boldsymbol{r}}|) P_{\mathfrak{n}}\left(\tilde{\boldsymbol{r}}-\boldsymbol{R}_{I}\right) \\
& +\iint d \boldsymbol{r} d \tilde{\boldsymbol{r}} \varphi_{i}\left(\boldsymbol{r}-\boldsymbol{R}_{I}\right) \varphi_{j}\left(\boldsymbol{r}-\boldsymbol{R}_{J}\right) v(|\boldsymbol{r}-\tilde{\boldsymbol{r}}|) \frac{\partial P_{\mathfrak{n}}\left(\tilde{\boldsymbol{r}}-\boldsymbol{R}_{I}\right)}{\partial R_{I, \lambda}} \\
= & -\iint d \boldsymbol{r} d \tilde{\boldsymbol{r}} \frac{\partial \varphi_{i}\left(\boldsymbol{r}-\boldsymbol{R}_{I}\right)}{\partial r_{\lambda}} \varphi_{j}\left(\boldsymbol{r}-\boldsymbol{R}_{J}\right) v(|\boldsymbol{r}-\tilde{\boldsymbol{r}}|) P_{\mathfrak{n}}\left(\tilde{\boldsymbol{r}}-\boldsymbol{R}_{I}\right) \\
& -\iint d \boldsymbol{r} d \tilde{\boldsymbol{r}} \varphi_{i}\left(\boldsymbol{r}-\boldsymbol{R}_{I}\right) \varphi_{j}\left(\boldsymbol{r}-\boldsymbol{R}_{J}\right) v(|\boldsymbol{r}-\tilde{\boldsymbol{r}}|) \frac{\partial P_{\mathfrak{n}}\left(\tilde{\boldsymbol{r}}-\boldsymbol{R}_{I}\right)}{\partial \tilde{r}_{\lambda}} \\
I P & \iint d \boldsymbol{r} d \tilde{\boldsymbol{r}} \varphi_{i}\left(\boldsymbol{r}-\boldsymbol{R}_{I}\right) \frac{\partial \varphi_{J}\left(\boldsymbol{r}-\boldsymbol{R}_{J}\right)}{\partial r_{\lambda}} v(|\boldsymbol{r}-\tilde{\boldsymbol{r}}|) P_{\mathfrak{n}}\left(\tilde{\boldsymbol{r}}-\boldsymbol{R}_{I}\right) \\
& +\iint d \boldsymbol{r} d \tilde{\boldsymbol{r}} \varphi_{i}\left(\boldsymbol{r}-\boldsymbol{R}_{I}\right) \varphi_{j}\left(\boldsymbol{r}-\boldsymbol{R}_{J}\right) \frac{\partial v(|\boldsymbol{r}-\tilde{\boldsymbol{r}}|)}{\partial r_{\lambda}} P_{\mathfrak{n}}\left(\tilde{\boldsymbol{r}}-\boldsymbol{R}_{I}\right) \\
& +\iint d \boldsymbol{r} d \tilde{\boldsymbol{r}} \varphi_{i}\left(\boldsymbol{r}-\boldsymbol{R}_{I}\right) \varphi_{j}\left(\boldsymbol{r}-\boldsymbol{R}_{J}\right) \frac{\partial v(|\boldsymbol{r}-\tilde{\boldsymbol{r}}|)}{\partial \tilde{r}_{\lambda}} P_{\mathfrak{n}}\left(\tilde{\boldsymbol{r}}-\boldsymbol{R}_{I}\right) \\
= & \iint d \boldsymbol{r} d \tilde{\boldsymbol{r}} \varphi_{i}\left(\boldsymbol{r}-\boldsymbol{R}_{I}\right) \frac{\partial \varphi_{J}\left(\boldsymbol{r}-\boldsymbol{R}_{J}\right)}{\partial R_{J, \lambda}} v(|\boldsymbol{r}-\tilde{\boldsymbol{r}}|) P_{\mathfrak{n}}\left(\tilde{\boldsymbol{r}}-\boldsymbol{R}_{I}\right) \\
= & -\frac{\partial(i(I) j(J) \mid \mathfrak{n}(I))}{\partial R_{J, \lambda}} .
\end{aligned}
$$

We have used that $\frac{\partial v(|\boldsymbol{r}-\tilde{\boldsymbol{r}}|)}{\partial r_{\lambda}}=-\frac{\partial v(|\boldsymbol{r}-\tilde{\boldsymbol{r}}|)}{\partial \tilde{r}_{\lambda}}$. From this result, we can derive the same relation if $\mathrm{n}$ is centered on atom $J$ by swapping $i(I)$ with $j(J)$ in the Coulomb integral which changes nothing per definition (Eq. (80) ) and then substituting the variable name 
$I$ with $J$ and $J$ with $I$, respectively, and the same for the variables $i$ and $j$. Hence, we obtain

$$
\frac{\partial(i(I) j(J) \mid \mathfrak{n}(J))}{\partial R_{I, \lambda}}=-\frac{\partial(i(I) j(J) \mid \mathfrak{n}(J))}{\partial R_{J, \lambda}} .
$$

Next, we consider the inverse Coulomb matrix $L^{I J}$. The derivative $\frac{\partial}{\partial X}$ of an inverse matrix $A^{-1}$ is given by $\frac{\partial A^{-1}}{\partial X}=$ $-A^{-1} \frac{\partial A}{\partial X} A^{-1}$. Additionally, we will need that the derivatives of the on-site terms of the Coulomb matrix vanish, i.e., $\frac{\partial V_{\mathrm{m}(I) \mathrm{n}(I)}}{\partial R_{I, I}}=$ 0 , which can be easily shown by using again integration by parts. However, this is not true for the inverse Coulomb matrix and it does not matter for the following derivation on which atom the auxiliary basis functions $m$ and $n$ are centered. With this said, the derivative of the inverse Coulomb matrix becomes

$$
\begin{aligned}
\frac{\partial L_{\mathfrak{r m}}^{I J}}{\partial R_{I, \lambda}}= & -\sum_{\mathfrak{r}, \mathfrak{s} \in \mathcal{P}(I J)} L_{\mathfrak{r r}}^{I J} \frac{\partial V_{\mathfrak{r} \mathfrak{s}}^{I J}}{\partial R_{I, \lambda}} L_{\mathfrak{s m}}^{I J} \\
= & -\sum_{\substack{\mathrm{r} \in \mathcal{P}(I) \\
\mathfrak{s} \in \mathcal{P}(J)}} L_{\mathfrak{r m}}^{I J} \frac{\partial V_{\mathrm{r}(I) \mathfrak{s}(J)}^{I J}}{\partial R_{I, \lambda}} L_{\mathfrak{s m}}^{I J} \\
& -\sum_{\substack{\mathfrak{r} \in \mathcal{P}(J) \\
\mathfrak{s} \in \mathcal{P}(I)}} L_{\mathfrak{r r}}^{I J} \frac{\partial V_{\mathrm{r}(J) \mathfrak{s}(I)}^{I J}}{\partial R_{I, \lambda}} L_{\mathfrak{s m}}^{I J} \\
= & \sum_{\substack{\mathrm{r}, \mathfrak{P} \in \mathcal{P}(I J)\\
}} L_{\mathfrak{r m}}^{I J} \frac{\partial V_{\mathfrak{r} \mathfrak{s}}^{I J}}{\partial R_{J, \lambda}} L_{\mathfrak{s m}}^{I J} \\
= & -\frac{\partial L_{\mathfrak{r m}}^{I J}}{\partial R_{J, \lambda}},
\end{aligned}
$$

where we have used Eq. (B.1).

Now, we have all the necessary relations and we can tend to the expansion coefficients using Eq. (B.2), B.3), and (B.4)

$$
\begin{aligned}
& \frac{\partial C_{i j}^{\mathfrak{m}}}{\partial R_{I, \lambda}}=\sum_{\mathfrak{n} \in \mathcal{P}(I J)}\left[\frac{\partial(i j \mid \mathfrak{n})}{\partial R_{I, \lambda}} L_{\mathfrak{n} \mathfrak{m}}^{I J}+(i j \mid \mathfrak{n}) \frac{\partial L_{\mathfrak{n}}^{I J}}{\partial R_{I, \lambda}}\right] \\
& =-\sum_{\mathfrak{n} \in \mathcal{P}(I J)}\left[\frac{\partial(i j \mid \mathfrak{n})}{\partial R_{J, \lambda}} L_{\mathfrak{n} \mathfrak{m}}^{I J}+(i j \mid \mathfrak{n}) \frac{\partial L_{\mathfrak{n} \mathrm{m}}^{I J}}{\partial R_{J, \lambda}}\right] \\
& =-\frac{\partial C_{i j}^{\mathrm{m}}}{\partial R_{J, \lambda}} .
\end{aligned}
$$

For the inverse Coulomb matrix $L_{\mathrm{nm}}^{I J}$, the change of the derivative does not depend on which atom the auxiliary basis functions $m$ and $n$ are centered. For the Coulomb integral $(i j \mid \mathfrak{n})$, there are only two possibilities, either $n$ is centered on atom $I$ or $J$. Both cases are covered by Eq. (B.2) and (B.3) and we can change the derivative.

\section{Appendix C. “Modified Stratmann” partition function}

Here, the partition function for integrals proposed by Stratmann and coworkers [46] is briefly reviewed and the modification of this approach that is implemented in FHI-aims is presented.
The goal is to find an expression for the function $g_{I}(\boldsymbol{r})$ in Eq. (111) that is strongly peaked around atom $I$ which allows fast and accurate numerical integration. For this purpose, confocal elliptical coordinates $\mu_{I J}(\boldsymbol{r})$ between pairs of atoms are defined [46],

$$
\mu_{I J}(\boldsymbol{r})=\frac{\left|\boldsymbol{r}-\boldsymbol{R}_{I}\right|-\left|\boldsymbol{r}-\boldsymbol{R}_{J}\right|}{\left|\boldsymbol{R}_{I}-\boldsymbol{R}_{J}\right|},
$$

where $\boldsymbol{R}_{I}$ and $\boldsymbol{R}_{J}$ are the positions of atom $I$ and atom $J$, respectively. The range of $\mu_{I J}$ is limited to $-1 \leq \mu_{I J}(\boldsymbol{r}) \leq 1$. Next a piecewise function $h$ is defined,

$$
h\left(\mu_{I J}, a\right)= \begin{cases}1, & \mu_{I J} \leq-a \\ k\left(\mu_{I J}, a\right), & -a<\mu_{I J}(\boldsymbol{r})<a \\ -1, & \mu_{I J} \geq a,\end{cases}
$$

using the threshold $a$. In practice, an empirically determined value of $a=0.64$ is used. The function $k$ is required to be continuously differentiable at $\mu_{I J}= \pm a$. The proposed form of Stratmann and coworkers for $k$ is

$$
k\left(\mu_{I J}, a\right)=\frac{1}{16}\left[35\left(\frac{\mu_{I J}}{a}\right)-35\left(\frac{\mu_{I J}}{a}\right)^{3}+21\left(\frac{\mu_{I J}}{a}\right)^{5}-5\left(\frac{\mu_{I J}}{a}\right)^{7}\right] .
$$

This defines the so-called cell function,

$$
s\left(\mu_{I J}, a\right)=\frac{1}{2}\left[1-h\left(\mu_{I J}, a\right)\right],
$$

the values of which are in the range between 0 and 1 . The function $g_{I}(\boldsymbol{r})$ is then given by

$$
g_{I}(\boldsymbol{r})=\prod_{J \neq I} s\left(\mu_{I J}(\boldsymbol{r}), a\right) .
$$

Despite the threshold $a$ in Eq. (C.2), the distance up to which an atom contributes to the cell function can be very large. This poses a problem for periodic systems because it would lead to large integration volumes. In order to solve this problem, the partition function by Stratmann et al. as described above has been modified inside FHI-aims. Only atoms which are closer to the integration point $\boldsymbol{r}$ than the maximal extend of their associated basis functions are allowed to contribute. In the case of the numeric atom-centered orbitals of FHI-aims, the maximal extend is given by the cut-off radius $r_{\text {cut }}$ of the confining potential $v_{\text {cut }}$ (see Eq. (9) in Ref. [16]). A typical value of $r_{\text {cut }}$ is $6 \AA$. To avoid discontinuities, the following interpolation scheme for the cell function $s$ of Eq. C.4 is employed:

$$
s_{\bmod }\left(\mu_{I J}(\boldsymbol{r}), a\right)=\left(1-u_{J}(\boldsymbol{r})\right)+u_{J}(\boldsymbol{r}) s\left(\mu_{I J}(\boldsymbol{r}), a\right)
$$

with

$$
u_{J}(\boldsymbol{r})= \begin{cases}1, & d_{J} \leq b r_{\text {cut }}^{J} \\ v_{J}(\boldsymbol{r}), & b r_{J}^{\mathrm{cut}}<d_{J}<r_{\text {cut }}^{J} \\ 0, & d_{J} \geq r_{\text {cut }}^{J}\end{cases}
$$

and

$$
v_{J}(\boldsymbol{r})=\frac{1}{2}+\frac{1}{2} \cos \left[\pi \frac{d_{J} / r_{\mathrm{cut}}^{J}-b}{1-b}\right] .
$$

Here, $d_{J}=\left|\boldsymbol{r}-\boldsymbol{R}_{J}\right|$ is the distance between integration point $\boldsymbol{r}$ and atom $J$. The transition threshold $b$ has been chosen as $b=$ 0.8. In summary, this modification allows to use the Stratmann partition function together with a restricted atom list without introducing any discontinuities. 


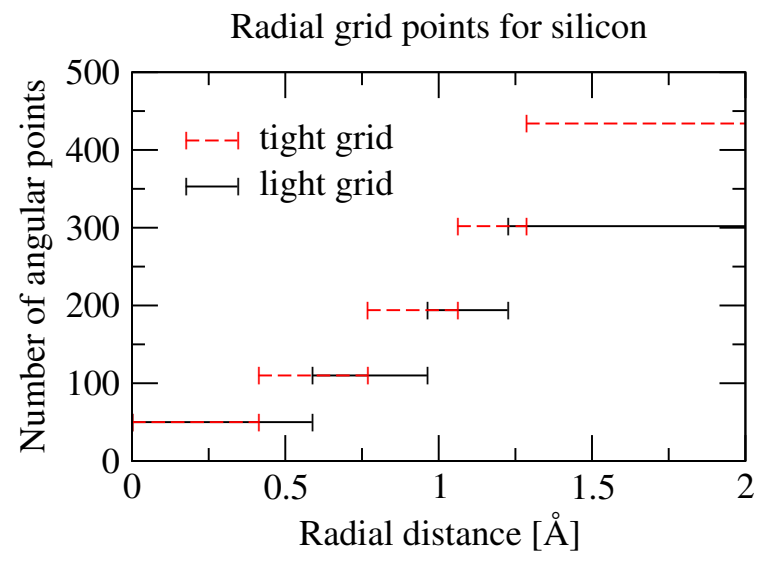

Figure D.13: Number of angular integration points for silicon in dependence of the radial distance from the nucleus. For the tight integration grid (red, dashed), the number of points increases faster with increasing distance and is overall larger in the most distant region in comparison to the light integration grid (black, solid). The number of integration points stays constant beyond $2 \AA$.

Integration shells for silicon $-\mathrm{N}_{\mathrm{r}}=42$

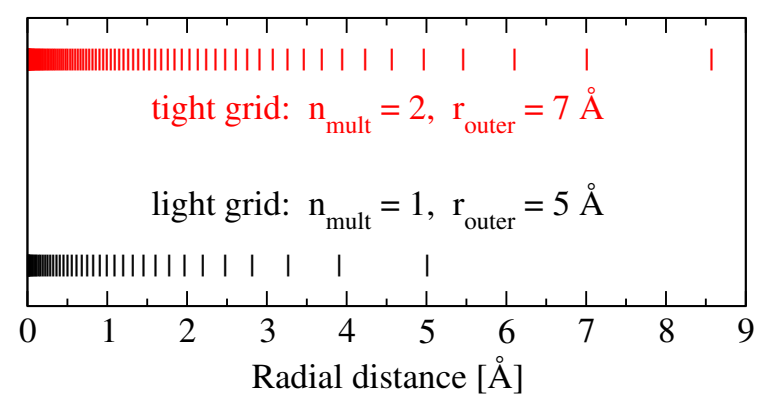

Figure D.14: Spherical integration shells for silicon. The position of these shells around an atom is shown and they are determined by Eq. (D.1). The light integration grid (black, bottom) uses a radial multiplier $n_{\text {mult }}=1$ and an extension of the shells $r_{\text {outer }}=5 \AA$. For the tight integration grid (red, top) the values are $n_{\text {mult }}=2$ and $r_{\text {outer }}=7 \AA$.

\section{Appendix D. Numerical settings}

\section{Appendix D.1. Integration grid}

FHI-aims performs the numerical integration on overlapping, atom-centered grids (details are described in Ref. [16]). Each integrand is divided into localized atom-centered pieces (see Eq. (111) ) and each of these pieces is then integrated on its own grid. An atom-centered grid consists of $N_{r}$ spherical integration shells $r(s)\left(s=1, \ldots, N_{r}\right)$ which are centered around the corresponding atom and extend up to an outermost shell at distance $r_{\text {outer }}$ from the nucleus. As proposed by Baker et al. [49], the positions of the integration shells are determined by

$$
r(s)=r_{\text {outer }} \frac{\log \left[1-\left(s /\left(N_{\mathrm{r}}+1\right)^{2}\right)\right]}{\log \left[1-\left(N_{\mathrm{r}} /\left(N_{\mathrm{r}}+1\right)^{2}\right)\right]} .
$$

Angular integration points are distributed on each shell such that spherical harmonics up to a certain order are integrated exactly. This kind of grid is called Lebedev grid and a version proposed by Delley [50] is employed. More angular integration points are needed for far away shells than for those close to the
Table D.4: Integration grids of silicon. The specification of the light and tight integration grid are shown here. $N_{r}$ specifies the number of spherical integration shells around each atom, $r_{\text {outer }}$ the extend of the grid, $n_{\text {mult }}$ the radial multiplier, $N_{\text {ang }}$ the number of angular integration points, and $N_{\text {tot,atom }}$ the total number of grid points per atom. See also text.

\begin{tabular}{lcc}
\hline & light grid & tight grid \\
\hline$N_{r}$ & 42 & 42 \\
$r_{\text {outer }}$ & $5 \AA$ & $7 \AA$ \\
$n_{\text {mult }}$ & 1 & 2 \\
$\min \left(N_{\text {ang }}\right)$ & 50 & 50 \\
$\max \left(N_{\text {ang }}\right)$ & 302 & 434 \\
$N_{\text {tot,atom }}$ & 5604 & 17918 \\
\hline
\end{tabular}

Table D.5: Shorthand notation for the parameters used to obtain the radial functions for silicon according to Eq. (8) in Ref. [16]. For the confinement potential $v_{\text {cut }}$, we used $r_{\text {onset }}=4 \AA$ and $w_{\text {cut }}=2 \AA$ (see Eq. (9) in Ref. [16]). The minimal basis consist of the radial functions of the occupied orbitals of spherically symmetric free atoms with noble gas configuration and quantum numbers of the additional valence functions. $\mathrm{H}(n l, z)$ denotes a hydrogen-like radial function for the Coulomb potential $z / r$ with radial and angular quantum numbers $n$ and $l$, respectively. $\mathrm{X}^{2+}(n l)$ denotes a $n, l$ function for the doubly positive charged ion of element X. See Ref. [16] for more details.

\begin{tabular}{ll}
\hline & $\mathrm{Si}$ \\
\hline minimal & {$[\mathrm{Ne}]+3 \mathrm{~s} 3 \mathrm{p}$} \\
tier 1 & $\mathrm{H}(3 d, 4.2)$ \\
& $\mathrm{H}(2 p, 1.4)$ \\
& $\mathrm{H}(4 f, 6.2)$ \\
& $\mathrm{Si}^{2+}(3 s)$ \\
tier 2 & $\mathrm{H}(3 d, 9.2)$ \\
& $\mathrm{H}(5 g, 9.4)$ \\
& $\mathrm{H}(4 p, 4.0)$ \\
& $\mathrm{H}(1 s, 0.65)$ \\
tier 3 & $\mathrm{Si}{ }^{2+}(3 d)$ \\
& $\mathrm{H}(3 s, 2.6)$ \\
& $\mathrm{H}(4 f, 8.4)$ \\
& $\mathrm{H}(3 d, 3.4)$ \\
& $\mathrm{H}(3 p, 7.8)$ \\
\hline
\end{tabular}

nucleus. Fig. D.13 shows exemplarily the number of points as a function of the radial distance for silicon. Adding up the angular integration points of all shells, gives the total number of

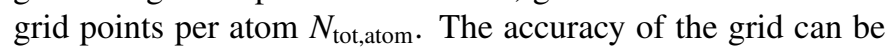
increased uniformly by placing additional shells at integer fractions of the original grid. The radial multiplier $n_{\text {mult }}$ denotes the denominator of these fractions. E.g., a radial multiplier of 2 places additional shells at $s=\frac{1}{2}, s=\frac{3}{2}, \ldots, s=N_{r}+\frac{1}{2}$, resulting in $2 N_{r}+1$ total shells. Fig. D.14 shows exemplarily the position of the integration shells for different $n_{\text {mult }}$ and $r_{\text {outer }}$ in the case of silicon. In Tab. D.4 the specifications of the light and tight integration grid for silicon are shown which were used for the study of the numerical correction terms.

\section{Appendix D.2. Basis set}

The atom-centered basis set of FHI-aims (details are described in Ref. [16]) is defined by the numerically tabulated ra- 
dial functions. Variational flexibility of this basis set is achieved by successively adding different radial functions. For each chemical element, radial functions are grouped in so-called tiers, namely "tier 1", "tier 2", and so on. The successive basis sets are hierarchical. For instance, "tier 1" for silicon consists of a $s, p, d, f$ radial function each, as specified in Tab. D.5. A "tier 2" basis set adds further radial functions while including the radial functions of "tier 1" as well. Tab. D.5 shows the shorthand notation for the parameters used to obtain the radial functions of silicon for those basis sets which were used for the study of the analytical stress for different basis set sizes.

\section{References}

[1] X. Gonze, J.-P. Vigneron, Phys. Rev. B 39 (1989) 13120-13128. doi 10. 1103/PhysRevB.39.13120

[2] P. Pulay, Mol. Phys. 17 (1969) 197-204. doi 10.1080/ 00268976900100941

[3] K. Reuter, C. Stampfl, M. Scheffler, in: S. Yip (Ed.), Handbook of Materials Modeling, Springer Netherlands, 2005, pp. 149-194. doi 10.1007/ 978-1-4020-3286-8

[4] J. F. Nye, Physical Properties of Crystals: Their Representation by Tensors and Matrices, Oxford University Press, 1985.

[5] N. Nagasako, T. Oguchi, J. Phys. Soc. Japan 80 (2011) 024701. doi 10. $1143 /$ JPSJ.80.024701

[6] O. H. Nielsen, R. M. Martin, Phys. Rev. Lett. 50 (1983) 697-700. doi 10. 1103/PhysRevLett.50.697

[7] O. H. Nielsen, R. M. Martin, Phys. Rev. B 32 (1985) 3792-3805. doi 10. 1103/PhysRevB.32.3792

[8] P. Focher, G. L. Chiarotti, in: L. Reatto, F. Manghi (Eds.), Progress in Computational Physics of Matter, World Scientific, 1995, pp. 1-42. doi $10.1142 / 9789814261319 \backslash 0001$

[9] T. Thonhauser, C. Ambrosch-Draxl, D. J. Singh, Solid State Commun. 124 (2002) 275-282. doi 10.1016/S0038-1098(02) 00181-3

[10] G. Kresse, D. Joubert, Phys. Rev. B 59 (1999) 1758-1775. doi 10.1103/ PhysRevB.59.1758

[11] M. Torrent, F. Jollet, F. Bottin, G. Zérah, X. Gonze, Comput. Mater. Sci. 42 (2008) 337-351. doi 10.1016/j . commat sci.2007.07.020

[12] K. N. Kudin, G. E. Scuseria, Phys. Rev. B 61 (2000) 16440-16453. doi 10.1103/PhysRevB.61.16440

[13] K. Doll, R. Dovesi, R. Orlando, Theor. Chem. Acc. 112 (2004) 394-402. doi $10.1007 / \mathrm{s} 00214-004-0595-\mathrm{y}$

[14] K. Doll, Mol. Phys. 108 (2010) 223-227. doi 10.1080/ 00268970903193028

[15] J. M. Soler, E. Artacho, J. D. Gale, A. García, J. Junquera, P. Ordejón, D. Sánchez-Portal, J. Phys. Condens. Matter 14 (2002) 2745-2779. doi $10.1088 / 0953-8984 / 14 / 11 / 302$

[16] V. Blum, R. Gehrke, F. Hanke, P. Havu, V. Havu, X. Ren, K. Reuter, M. Scheffler, Comput. Phys. Commun. 180 (2009) 2175-2196. doi 10 . $1016 / j . c p c .2009 .06 .022$

[17] A. Tkatchenko, M. Scheffler, Phys. Rev. Lett. 102 (2009) 073005. doi 10. 1103/PhysRevLett.102.073005

[18] B. Delley, J. Phys. Chem. 100 (1996) 6107-6110. doi 10.1021/ jp952713n

[19] P. Hohenberg, W. Kohn, Phys. Rev. 136 (1964) B864-B871. doi 10. 1103/PhysRev.136.B864

[20] W. Kohn, L. J. Sham, Phys. Rev. 140 (1965) A1133-A1138. doi 10. 1103/PhysRev.140.A1133

[21] B. Delley, J. Chem. Phys. 92 (1990) 508. doi 10.1063/1.458452

[22] A. D. Becke, R. M. Dickson, J. Chem. Phys. 89 (1988) 2993. doi 10. $1063 / 1.455005$

[23] B. I. Dunlap, J. W. D. Connolly, J. R. Sabin, J. Chem. Phys. 71 (1979) 3396. doi $10.1063 / 1.438728$

[24] S. H. Vosko, L. Wilk, M. Nusair, Can. J. Phys. 58 (1980) 1200-1211. doi $10.1139 / \mathrm{p} 80-159$

[25] J. P. Perdew, A. Zunger, Phys. Rev. B 23 (1981) 5048-5079. doi 10. 1103/PhysRevB.23.5048
[26] J. P. Perdew, Y. Wang, Phys. Rev. B 45 (1992) 13244-13249. doi 10. 1103/PhysRevB.45.13244

[27] J. P. Perdew, K. Burke, M. Ernzerhof, Phys. Rev. Lett. 77 (1996) 38653868. doi 10.1103/PhysRevLett.77.3865

[28] A. D. Becke, J. Chem. Phys. 88 (1988) 1053. doi 10.1063/1.454274

[29] C. Lee, W. Yang, R. G. Parr, Phys. Rev. B 37 (1988) 785-789. doi 10. 1103/PhysRevB.37.785

[30] A. D. Becke, J. Chem. Phys. 98 (1993) 1372. doi 10.1063/1.464304

[31] A. D. Becke, J. Chem. Phys. 98 (1993) 5648. doi 10.1063/1.464913

[32] J. P. Perdew, M. Ernzerhof, K. Burke, J. Chem. Phys. 105 (1996) 9982. doi $10.1063 / 1.472933$

[33] J. Heyd, G. E. Scuseria, M. Ernzerhof, J. Chem. Phys. 118 (2003) 8207. doi $10.1063 / 1.1564060$

[34] J. Heyd, G. E. Scuseria, M. Ernzerhof, J. Chem. Phys. 124 (2006) 219906. doi $10.1063 / 1.2204597$

[35] A. V. Krukau, O. A. Vydrov, A. F. Izmaylov, G. E. Scuseria, J. Chem. Phys. 125 (2006) 224106. doi 10.1063/1.2404663

[36] X. Ren, P. Rinke, V. Blum, J. Wieferink, A. Tkatchenko, A. Sanfilippo, K. Reuter, M. Scheffler, New J. Phys. 14 (2012) 053020. doi 10.1088/ $1367-2630 / 14 / 5 / 053020$

[37] C. Van Alsenoy, J. Comput. Chem. 9 (1988) 620-626. doi 10.1002/ jcc.540090607

[38] O. Vahtras, J. Almlöf, M. W. Feyereisen, Chem. Phys. Lett. 213 (1993) 514-518. doi 10.1016/0009-2614(93)89151-7

[39] A. C. Ihrig, J. Wieferink, I. Y. Zhang, M. Ropo, X. Ren, P. Rinke, M. Scheffler, V. Blum, in preparation.

[40] FHI-aims release Aug 2013.

[41] R. Ahlrichs, R. Penco, G. Scoles, Chem. Phys. 19 (1977) 119-130. doi 10.1016/0301-0104(77) 85124-0

[42] Q. Wu, W. Yang, J. Chem. Phys. 116 (2002) 515. doi 10.1063/1. 1424928

[43] S. Grimme, J. Comput. Chem. 25 (2004) 1463-1473. doi 10.1002/jcc. 20078

[44] T. Bučko, S. Lebègue, J. Hafner, J. G. Ángyán, Phys. Rev. B 87 (2013) 064110. doi 10.1103/PhysRevB.87.064110

[45] R. Golesorkhtabar, P. Pavone, J. Spitaler, P. Puschnig, C. Draxl, Comput. Phys. Commun. 184 (2013) 1861-1873. doi 10.1016/j.cpc.2013. 03.010

[46] R. E. Stratmann, G. E. Scuseria, M. J. Frisch, Chem. Phys. Lett. 257 (1996) 213-223. doi 10.1016/0009-2614(96)00600-8

[47] R. Lindh, A. Bernhardsson, G. Karlström, P.-A. Malmqvist, Chem. Phys. Lett. 241 (1995) 423-428. doi 10.1016/0009-2614(95) 00646-L

[48] V. Bakken, T. Helgaker, J. Chem. Phys. 117 (2002) 9160. doi 10.1063/ 1.1515483

[49] J. Baker, J. Andzelm, A. Scheiner, B. Delley, J. Chem. Phys. 101 (1994) 8894. doi $10.1063 / 1.468081$

[50] B. Delley, J. Comput. Chem. 17 (1996) 1152-1155. doi 10.1002/ (SICI) 1096-987X (19960715) 17:9<1152: :AID-JCC7>3.0.CD; $2-R$ 